\title{
The current state of high-fidelity simulations for main gas path turbomachinery components and their industrial impact
}

\author{
Richard D. Sandberg • Vittorio Michelassi
}

Received: date / Accepted: date

\begin{abstract}
Over the past two decades high-fidelity simulations have become feasible for most main gas path turbomachinery components. This paper introduces the key challenges of simulating and modelling turbomachinery flows and presents an overview of possible simulation strategies. A comprehensive overview is given of which particular design challenges have to date been addressed by high-fidelity simulations, with a particular focus on high-pressure and centrifugal compressors, and high-pressure and low-pressure turbines. Recommendations are provided for how quality and accuracy can be ensured for high-fidelity simulations, using direct numerical simulations of a low-pressure turbine as a case study. It is argued that industrial impact from high-fidelity simulations can be achieved in two ways, either by conducting design-of-experiment-like studies that can provide designers with insight into certain physical mechanisms and phenomena, or by helping mature and improve lower-order models. The latter is discussed with particular emphasis on recent advances in machine learning for model assessment and improvement, and the potential of one selected data-driven approach is demonstrated on predictions of wake mixing for low-pressure and high-pressure turbines.
\end{abstract}

Keywords High-fidelity simulations · turbomachinery · DNS · LES · machine learning

\section{Introduction}

Turbomachines, ranging from gas to steam turbines, pumps and compressors are used in a very wide range of applications. Turbines are used for the generation of mechanical power, eventually converted into electricity, and for thrust generation in aircraft

R. D. Sandberg

Department of Mechanical Engineering, University of Melbourne, Australia

E-mail: richard.sandberg@unimelb.edu.au

V. Michelassi

Baker Hughes, a GE company, Florence, Italy 
engines. Pumps and compressors find application in the chemical and petrochemical industries and for oil and gas distribution through pipelines. In particular, gas turbines $(G T)$ are an efficient and relatively clean way to convert chemical energy from fossil fuels into mechanical power and thrust, while steam turbines $S T$ are necessary to convert nuclear, thermal solar, or geothermal energy into mechanical or electrical power. Although wind turbines are often not considered turbomachines, they share some of the design challenges of $G T$ and $S T$. Recent $G T$ aeroderivative designs are also particularly suited for power generation in grids with a large contribution from renewable sources, like wind and solar, the intermittent nature of which calls for fastreacting and short start-up time power generation systems based on fossil fuels. In summary, whenever chemical, nuclear, geothermal, solar, or wind energy needs to be converted into power a turbomachine is involved (with the only exception of direct solar-to-electricity conversion). Aircraft propulsion is probably the fastest growing segment for $G T$, and it is also the application that calls for the highest technology content. The good efficiency and the high power density are among the main reasons of success of $G T$ for both narrow and wide body aircraft propulsion. It is instructive to realize that the US alone consumed approximately 35.6 billion gallons of aviation fuel in 2012 (FAA, 2014). As described by Laskowski et al. [58], the efficiency of a turbomachine in converting chemical energy into power depends on thermodynamic, aerodynamic, and thermal efficiency, while additional propulsion efficiency comes into play for aircraft engines only. In particular, the thermodynamic efficiency of the cycle is proportional to the pressure ratio, but high pressure ratios come at the expense of aerodynamic efficiency and operability range. In addition, power density can be obtained by increasing the firing temperature, but the aero-thermal efficiency of high-pressure-turbines will decrease due to cooling requirements. Overall, the design of high-efficiency GT needs to reconcile many different requirements and constraints, and the accuracy of aero-thermal design methods is of paramount importance.

Today, the aero-thermal-mechanical design of any turbomachine is heavily assisted by computational methods, which have a direct impact on the prediction of the different efficiencies mentioned above. Therefore, the accuracy of computational design methods must be put in the correct industrial perspective. The inaccurate prediction of the thermal load, aerodynamic efficiency, operability, and durability of a turbomachine component may require extremely expensive redesign and re-tests, sometimes worth hundreds of million dollars, without considering the delay in taking advantage of market and revenue opportunities. A simple way to address inaccurate design predictions would be to take so-called "engineering safety margins", for example downgrading expected performance. This is not acceptable as turbomachines are selected also on their return-of-investment time. Therefore, a turbomachine for which the efficiency is quoted low is expected to burn more fuel, or generate less power, and it will not be selected as its return-of-investment time will increase. Hence, design methods are asked to be more and more accurate.

A good example of design accuracy impact is given by the high-pressure turbine $(H P T)$ of a $G T$, that operates at high pressures and temperatures often at, or above, the material melting point. The mechanical integrity and the life of the HPT is guaranteed by the internal and external cooling system that cools the parts, and creates a thermal shield around vanes and blades. The HPT cooling air is extracted from 
the GT compressor and pumped into the HPT bypassing the combustion chamber. A design that could safely reduce by $1 \%$ the cooling air flow rate will increase by $0.4 \%$ the $G T$ cycle efficiency, depending on the $G T$ thermodynamic cycle. Another interesting example are compressors, needed to deliver pressurized air into the $G T$ combustion chamber. The aerodynamic efficiency of the compressor is critical to the energy conversion system quality, as $1 \%$ of compressor adiabatic efficiency can be worth $0.5 \%$ of cycle efficiency. It is therefore needless to say that the accuracy of both the cooling design and compressor design tools will need to be very high to appreciate less than $1 \%$ performance derivatives. The list could be easily extended to propulsion fans of high by-pass ratio aircraft engines, power turbines applied to both propulsion and mechanical drive engines, steam turbines and wind turbines. The impact of $G T$ component efficiency on the overall energy conversion efficiency may appear small, but the combined effects from different components can sum up to numbers exceeding multiple percentage points. Considering the huge turbomachinery installed base, including industrial, power generation and propulsion, even a small increase in the energy conversion cycle efficiency may result in multi-billion-dollar fuel burn savings and emission reduction.

The current $G T$ generation is the result of more than six decades of designs. As a result, $G T$ are already extremely reliable and efficient, and the associated technology fairly mature. Hence, it has become increasingly difficult to extract additional tenthof-point of performance or durability improvements, partly on account of the difficulties current design methods and performance verification tools have to guarantee the necessary degree of accuracy. Along these lines, the fundamental and industrial research aimed at improving energy conversion cycle efficiency spans from materials to engine architectures, manufacturing techniques, aerodynamics, thermodynamics, aeromechanics only to name a few. In particular, aerodynamics is important to determine compressor, combustor, and turbine efficiency, operability and durability and, ultimately, cost. The design of these three components is very heavily assisted by Computational Fluid Dynamics $(C F D)$. Unfortunately, CFD does not always have the required degree of accuracy in capturing the flow physics due to a number of challenges related to the flow complexity. One of the top offenders contributing to lack of CFD accuracy is the stochastic (turbulence driven) and deterministic (statorrotor interaction driven) nature of flow unsteadiness in turbomachinery, with the two phenomena generally featuring different frequencies. The interaction of the two phenomena drives the mixing process of momentum and enthalpy which, in turn, controls the growth of irreversibilities and ultimately efficiency. Despite the difficulties caused by some well documented inaccuracies, design iterations are based upon ReynoldsAveraged Navier-Stokes methods, RANS, or their unsteady counter part, unsteady RANS, URANS, in which all scales of turbulence are modelled. The inaccuracies, some of which were illustrated by Pichler et al. [104] do not allow a fully reliable design optimization and technology maturation.

Here Direct Numerical Simulation (DNS) and Large Eddy Simulation (LES) come into play as they both resolve the turbulent unsteady flow field. DNS and LES have been proven able to reproduce turbomachinery flow physics with high degree of accuracy. Nevertheless, the associated computational cost, more than $100 \times$ with respect to conventional RANS and URANS, make them practically inapplicable to 
perform design iterations (e.g. [86, 117, 152]) even when focussing on simplified geometries. It has been shown in some cases, though, that adopting coarse grids to run Very Large Eddy Simulations can be sufficiently affordable to enable genetic-based optimizations of specific turbine aerodynamic features (e.g. [147]).

Another important factor that increases the overall computational cost is the stator and rotor blade count that, in addition to aerodynamic requirements, is determined such that aeromechanics issues like forced response and high-cycle fatigue are avoided. Stator and rotor count are often prime numbers for both turbines and compressors so that single and multi-stage simulations would require a full 360-degree computational domain. While this is sometimes affordable in URANS, it is currently not possible using DNS and LES. It is thus necessary to approximate the real statorrotor count by adjusting pitch and/or chord to limit the number of row passages to $1-2$, or a maximum of $2-3$, where the first and second number refer to the stator and rotor passages, respectively. The impact of such approximations must be carefully evaluated prior to embarking in very costly simulations, as it changes frequencies and throat areas that are two key design parameters.

Measurements can give important insights into $G T$ flow fields, but they are often expensive and can be difficult at realistic operating conditions, i.e. high pressure and temperature. Moreover, the flow details needed to understand the nature of the unsteady flow phenomena are only rarely available. In practice, a $G T$ designer must incorporate safety margins to the CFD results to ensure performance and component life meet expectations to compensate for the CFD assisted design loop aero-thermal loading prediction uncertainties. Current methods do not have sufficient accuracy to appreciate the flow physics details and the associated performance to drive a reliable design optimization. However, high-fidelity CFD, coupled with appropriate validation, provides an opportunity to resolve the physics in gas turbines with the required level of detail to develop advanced technologies.

With this in mind, this paper aims at reviewing the current state of high-fidelity simulations for turbomachinery applications and their impact on industry as a viable tool to assist the aero-thermal design of $G T$.

\section{The challenge of simulating turbulent flows}

It is well known that turbomachinery flows have both deterministic and stochastic contributions, the former being typically driven by stator-rotor interaction, while the latter being due to turbulence. These two distinct contributions are superposed onto a time-averaged flow field that can be subsonic or supersonic with or without shocks. What is less commonly understood is that the deterministic and stochastic unsteady components do interact, and it is their interaction that controls the majority of irreversibilities. From the standpoint of turbulence models based upon the Reynolds averaging approach, such interaction poses additional challenges that can be summarized in the spectral-gap problem. The time averaging process conceived by Reynolds assumes a shape of the energy content associated with the turbulent motion, but it does not necessarily include the additional contribution of deterministic unsteadiness. If the frequency associated with the deterministic contribution is lower than the 
frequencies associated with the turbulent energy cascade, as illustrated by the grey box labelled $B P F_{L}$ in figure 1, the Reynolds averaging process holds valid. In this situation there exists a measurable gap between the deterministic and the stochastic spectra than prevents any significant interaction between the two.

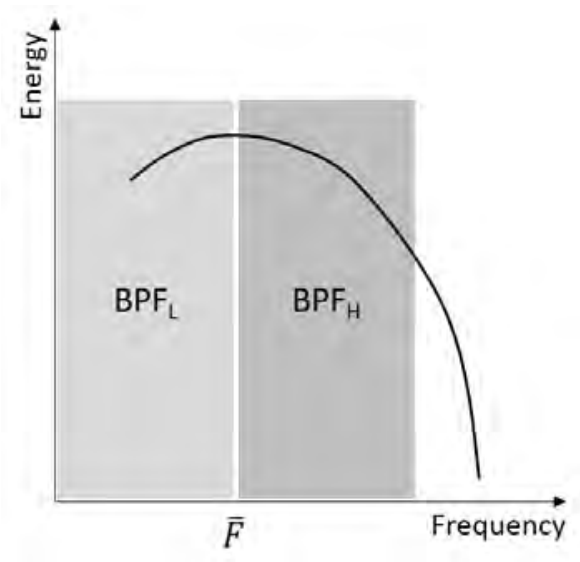

Fig. 1 Sketch of turbulent spectrum with blade passing frequency $(B P F)$ ranges

However, when the frequency of the deterministic unsteadiness is high and it overlaps with the frequencies associated with the large turbulent scales, as illustrated by the dark grey box labelled $B P F_{H}$ in figure 1 the spectral gap vanishes and the two spectral contributions interact. In the absence of the spectral gap the Reynolds averaging process does not hold valid any longer as it is not meant to model the concerted contribution of two unsteady phenomena, a deterministic and a stochastic one. This concept is described by Tucker [140] and by Michelassi [83] who suggest that in such flows a two-equation turbulence model will not perform as expected. Turbomachines are now smaller and the components rotate faster to reduce weight and increase power density. Therefore the frequencies and the harmonics associated with stator-rotor interaction are growing to the point that the spectral gap becomes insufficient to prevent the stochastic-deterministic interaction, for which conventional two equation models are not derived. Moreover, the improvements in manufacturing techniques and materials have allowed to safely reduce profile and trailing edge thicknesses, so for the same Strouhal number, the shedding frequencies increase to the point where they can interact with the turbulent spectrum. The increase in speed also comes with flow regimes that are moving from high-subsonic to transonic and supersonic. In such conditions, shocks and their interaction with endwall boundary layers become of paramount importance as a source of additional losses and flow instabilities. These are additional features that conventional turbulence models struggle to predict. Thus, the greatest challenge for accurate simulations of main gas-path flows in turbomachinery is the presence of turbulence and its interaction with deterministic unsteadiness, and in some cases the laminar-turbulent transition. 
Another fundamental difficulty in computing the internal flow in gas turbine components is the presence of curvature and pressure gradients. Curvature provokes a redistribution of turbulent shear stresses from a simple shear layer that is responsible for additional momentum and enthalpy diffusion and consequently entropy production that ultimately controls the onset of irreversibilities. But while curvature effects are common in turbulent flows, what characterize turbomachinery flows are the very large pressure gradients. With the improvements in materials, aeromechanics, and aerodynamics, turbines and compressors are subject to very strong favorable pressure gradients, like in the first half of high pressure turbine vanes and blades, in highly loaded low pressure turbines, and on the pressure side while approaching the trailing edge. Also, the strength of adverse pressure gradients, that need to be carefully controlled to avoid excessive growth of losses or the onset of separations, is growing, as witnessed by the reduced number of axial compressor stages for increasingly large pressure ratios. Such pressure ratios are responsible for massive deformation of the turbulent flow field, and they are generally beyond the range of validity of the original calibration and validation of two-equation models.

The flow in turbines and compressors is wall driven, as opposed to combustion chambers where the flow is wall bounded. This makes boundary layers very important in the former, as more that $80 \%$ of the losses are generated in the proximity of the boundary layer. It is nevertheless very instructive to observe that despite the strong background turbulence intensity grown by the interaction between stators with wakes and pressure waves, that can be larger than 5\%, turbulent boundary layers can relaminarize due to very strong accelerations, like on the pressure side trailing-edge region of turbines, from the leading edge to the peak suction of low pressure turbines and, under certain conditions, in the first half of axial compressor airfoils.

To summarize, the stochastic and deterministic nature of the flow, the presence of strong curvature, in conjunction with large favorable and adverse pressure gradients, and large turbulence intensities are the main difficulties encountered in the simulation of $G T$ flows.

\section{Simulation and modelling strategies}

As described in the previous paragraph, the unsteadiness of turbomachinery flows is comprised of random and periodic components. These two sources of unsteadiness must be addressed differently.

With reference to figure 2, the fine scale turbulent motion can be resolved only by conducting first-principle based simulations, i.e. directly solving the Navier-Stokes equations with so-called direct numerical simulations (DNS). The associated computational effort depends on the Reynolds number, $R e$, as it determines the size of the smallest length and time scales. It is possible to estimate the computational effort as a function of Re starting from an equilibrium flow. In one spatial direction the number of grid points required to resolve the smallest scale of turbulence motion is dictated by ratio of the Kolmogorov scale $\eta_{K}$ and the length of the largest scales $\ell$, associated with the geometry of the flow. The required grid nodes scales as $N^{1 D}=\ell / \eta_{K} \propto R e_{t}^{\frac{3}{4}}$, with $R e_{t}$ denoting the turbulence Reynolds number. The 
three-dimensional nature of turbulence, not necessarily isotropic, calls for a number of grid nodes in $3 D$ proportional to $R e_{t}^{9 / 4}$. By considering also the need to resolve the time scale associated with the length scale, the total computational effort becomes proportional to $R e_{t}^{3}$. A more refined estimate by Choi and Moin [10] for evolving boundary layers over a flat plate gives $R e_{L_{x}}^{37 / 14}$. Despite this challenging scaling, the continuous growth of computational resources allowed the computation at practical Reynolds numbers first of canonical cases such as homogeneous isotropic turbulence [48], moving to turbulent channel flow [61] and finally to turbomachinery flows in realistic conditions [152].

Unfortunately, most of the GT main gas-path simulations have a domain size that is much larger than that of the boundary layer evolving on a blade, and, consequently length scales may be considerably larger than $R e_{L_{x}}$. For these reasons, DNS cannot be used to simulate flows with the necessary geometrical fidelity and extension. For a general review on DNS and its challenges, see Moin and Mahesh [92].

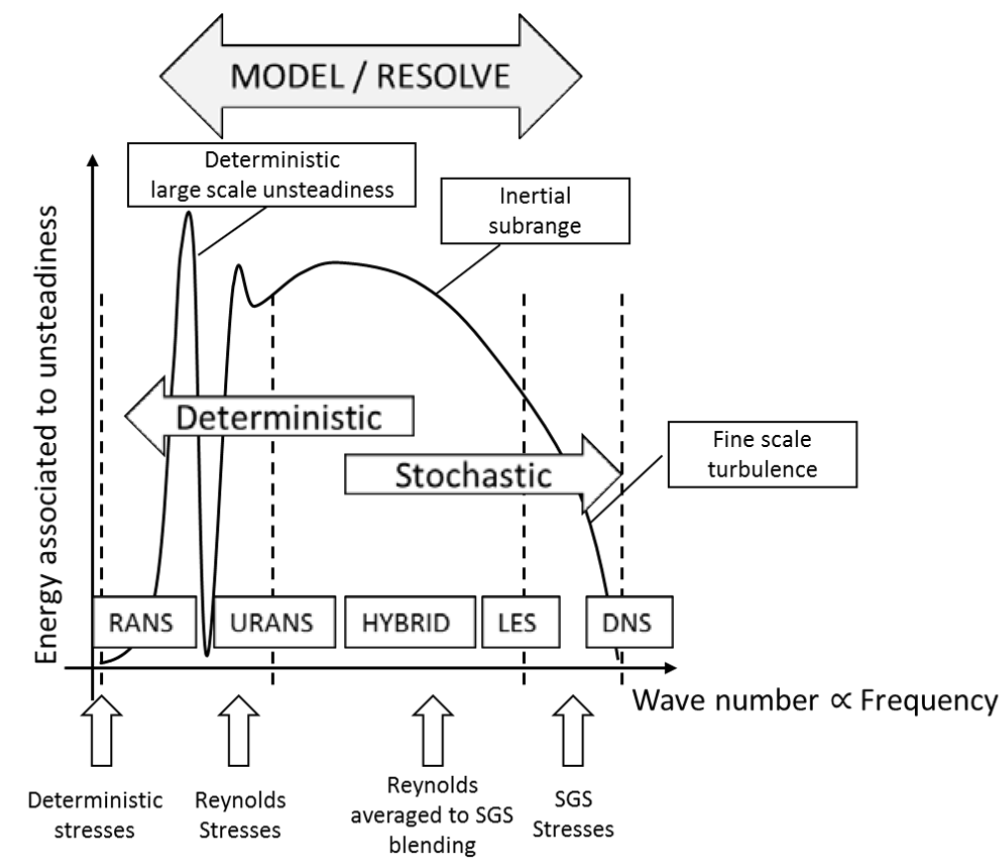

Fig. 2 Sketch of turbulence spectrum with turbulence model type as a function of cut-off frequency

Rather than resolving the fine scale turbulent motion, to the right of the inertial subrange in figure 2, it is possible to model them assuming they are substantially isotropic. Large Eddy Simulation (LES) does this by using a discretization in space and time unable to resolve the fine scale motions, and models the effect of these on the resolved part of the flow with a so-called sub-grid scale $(S G S)$ model. Such a model performs a local spatial averaging of the flow, as originally described by Smagorin- 
sky [127]. Successive evolutions of the $S G S$ model can be found in Germano [33], with further improvements to improve accuracy in wall driven flows [21]. The application of LES to turbomachinery flow has been quite successful, as described in the next sections, and it has allowed to shed some light on key physical phenomena in both turbines and compressors. However, LES still suffers in transitional boundary layers $[88,159]$. The $S G S$ model computes the unresolved sub-grid stresses by using local information only. Despite the large computational effort reduction over DNS (of the order of $10^{2}$ ), LES is not yet a viable design verification tool, or at least it cannot be practically used to perform hundreds of design iterations due to the size and complexity of the computational domain and the high $R e$.

The well known Reynolds averaging method, a much more drastic way to simplify turbulent flows, can be found in the leftmost section of figure 2. This approach requires the solution of the so-called Reynolds averaged Navier-Stokes (RANS) or unsteady RANS (URANS) for unsteady calculations that intend to resolve only the low frequencies, like those associated with a stator-rotor interaction. In these modified Navier-Stokes equations the averaging process causes two additional terms to appear, the turbulent stress and the turbulent heat flux, and require numerous assumptions to be made in order to close the problem. These two terms are diffusive in nature and their role is not really to model turbulence, rather to model the effect of turbulent momentum diffusion and turbulent enthalpy diffusion on the Reynolds averaged flow field. The gradient diffusion hypothesis is typically used to correlate these two terms to the local deformation of the time averaged velocity and thermal fields, respectively. The coefficient of proportionality between the turbulent shear stress tensor and the velocity deformation tensor is the so-called turbulence viscosity, that, with the additional contribution of the turbulent Prandtl number, is also the proportionality coefficient between the turbulent heat transfer vector and the time averaged temperature gradient vector. Therefore, after the gradient diffusion hypothesis is enforced, the only additional unknown needed to compute a time averaged flow field is the turbulence viscosity. Over more than 40 years a wide spectrum of models has been proposed. The simplest are local formulations that do not require any additional transport equation (similar to SGS models), like the well known BaldwinLomax [4], which is sometimes still used in the early stage of CFD assisted design iterations. In order to account for transport effects, one equation models, such as the Spalart-Allmaras model [132] or two-equation models were introduced. These latter models use a transport equation for the trace of the turbulent stress tensor, better known as turbulent kinetic energy, and an additional equation for either the turbulence frequency [153], or the turbulence dissipation [100], or the turbulent integral length scale as proposed by Rotta [115]. These models always determine a time and a length scale of turbulence to compute the turbulence viscosity, and require a computational effort that is a fraction of what is needed by LES, often more than a factor of 100. Despite some fundamental differences, like in the SST by Menter [80], such models always underpredict the turbulent diffusion of both momentum and enthalpy. As a consequence, steady and unsteady aerodynamic losses, as well as heat transfer and aerodynamic forcing for fluid-structure interaction, are not always predicted with the accuracy required to drive the evolution of the fairly mature aerothermal design of turbines and compressors. Such inaccuracy is driven by two distinct weaknesses: 
1 - The turbulence viscosity can be defined as the ratio of the turbulent stress tensor rank with the strain rate tensor rank. If a DNS flow field is available, such turbulence viscosity can be computed exactly, although its definition is not always straightforward [105, 155], and it can be compared with the turbulence viscosity coming from a two-equation model. The same can be done for the turbulent Prandtl number or directly for the turbulent heat flux coefficient [155]. The comparison reveals very large spatial differences between the two.

2 - Both turbulent stress and heat flux are tensors, and in principle one should verify not only the proportionality between the exact tensor and its modeled version, but also their alignment. Such alignment checks have been performed a number a times (e.g. $[86,87,135]$ and they have revealed that the concerted action of incoming wakes and pressure gradients can provoke a full misalignment.

In an attempt to cure some of the weaknesses described above, Gatski and Speziale [32] developed an evolution of the gradient diffusion hypothesis adding non-linear terms to the constitutive law that describes turbulent diffusion in RANS. After their original paper, new formulations, both quadratic and cubic, have been introduced to mitigate the numerical stiffness of non-linear RANS models. One of the most successful is the one of Wallin and Johansson [144], that proved measurable improvement with respect to linear laws. There is also a wide variety of models that sit between RANS and LES, like DES [128] and SAS [81]. These models blend RANS and LES depending on the local grid quality. Despite their attractiveness, the difficulty in defining a robust RANS-LES blend has to date limited their application for design verification.

\section{Review of recent high-fidelity simulation work and its industrial value}

The next sections will cover specific design challenges for each GT components and describe how scale-resolving simulations may assist in addressing those. The list is not supposed to be complete, but it addresses the major design uncertainties in which conventional CFD is known to fail.

\subsection{High-pressure Compressor}

Design Trends. High pressure compressors are probably among the most challenging components from the aerodynamic standpoint. Smith [129] offers a comprehensive summary of the difficulties and trends in axial compressor design for aero-propulsion. Although the paper dates back to 2002, the majority of the key questions still hold valid, as witnessed by the very wide literature on highly-loaded axial compressor aerodynamics [19]. Axial compressors design point performance is indeed of importance because if an engine experiences a compressor efficiency shortfall the user may need more fuel to get the same power. However, the operability range, i.e. the flow rate from choke to stall, is even more important as if the compressor is unstable, or if its operability range is too narrow, the engine cannot be started and/or operated. Therefore, on top of the design point, the aero design needs to address off-design performance as well. Moreover, some of the key points in axial compressor design are 
related to three-dimensional effects, stator-rotor interaction, off-design, and perturbations to the main flow fields, while the aerodynamics of a single compressor airfoil is relatively well understood. Before discussing in detail the fundamental investigations of axial compressors that have been conducted with high-fidelity simulations, it is worth observing how the engineering community is trying to take advantage of the improved accuracy of CFD, as discussed by Ivengar and Sankar [49]. It is foreseeable that such methodologies will be extended to LES or Hybrid-LES in the near future to address specific design topics in axial compressors. The compressor design trends are described in Smith [129] and Dickens and Day [19]

Advanced 3D shapes. Axial compressor stators and rotors are designed to balance the flow and pressure ratio distribution along the spanwise direction. Given the different relative speed from hub to tip, this often requires complex three-dimensional shapes, as described by McNulty et al. [77], who describe the impact of rotor blade tip forward sweep on performance and stall margin. Both measurements and CFD predictions suggest a very complex 3D flow pattern dominated by the streamwise vorticity generated in the rotor tip gap. The interaction of such secondary flow structures with the core flow ultimately governs the spanwise mass flow shift, the loss generation and the operability range. More recently, Taylor and Miller [138] further discussed how advanced three-dimensional rotor blades can help improving operability without jeopardizing efficiency. In particular, the combination of bowing and leaning proved very successful. At the same time, they also observed that CFD could be trusted only in a range of geometrical parameters that, once exceeded, caused CFD to significantly deviate from experiments. This may suggest a CFD confidence region that may limit the full exploitation of an optimal design. In summary, both investigations illustrated that RANS CFD was often able to capture the right trend, while flow details and absolute performance values were often not in agreement with experimental results. Moreover, with the growing geometrical complexity, RANS cannot reproduce flow details that may be essential for a proper design optimization.

Shock boundary-layer interaction. In the attempt to reduce their size, the tip speed of axial compressor front stages has increased to the transonic and supersonic flow regime. Hence, on top of the complexity of controlling a high-speed flow with adverse pressure gradients, the airfoil design must handle complex shock-boundary layer interactions in conjunction with aggressive profile cambering, while trying to avoid local stall. A compressor rotor blade shape optimization, aimed at reducing losses by minimizing the shock-boundary layer interaction, is described by Okui et al. [95], who used a multi-objective optimization method. The optimization, based on the response from RANS, produced complex three-dimensional shapes along the lines described by Taylor and Miller [138] and McNulty et al. [77]. Still, the complexity of the optimized geometry and the associated flow field raise some questions on the validity of the optimized solution. In particular, as demonstrated in Vieira and Azevedo [142], who compared four different turbulent closures, in presence of a strong shock boundary layer interaction RANS may not predict the flow correctly at the foot of the shock. Michelassi [82] showed that, while the shock strength and position may be captured correctly, the separation at the foot of a strong shock in presence of a downstream adverse pressure gradient was not captured accurately. 
Cavities and extractions. The adverse pressure gradient nature of the majority of the flow path amplifies the so-called secondary effects. It is well known that a jet in cross flow will provoke larger flow disruption if the core flow experiences an adverse pressure gradient and it does not benefit from the "polishing" effect of flow acceleration. The main compressor flow path is distorted by the presence of cavities at the interface between stator and rotor and small local leakages may act as a small jet released at an angle with respect to the core flow. Therefore, the interaction of the core flow with leakages alters the endwall boundary layers and may change local incidences and ultimately provoke anticipated stall, if not properly accounted for in the design phase. To make the picture even worse, axial compressors are equipped with flow extractions along the flow path for multiple purposes, as illustrated in figure 3. Such extractions are shaped like continuous slots or discrete holes, both at hub and shroud, to feed fresh air to the high-pressure turbine for cooling purposes, pressurize internal cavities to avoid hot gas ingestions, and facilitate start-up and shut-down of the compressor when the front and back stages are heavily mismatched. For example, during start-up when the rotor speed is low the front stages develop very low pressure ratio, but the volume flow is already high enough to choke the back-stages. To avoid these difficulties, compressors are also equipped with bleed ports, or with by-pass pipes, that divert the flow around half-way the compressor to reduce the volume flow of the back stages during start-up and avoid choking. Grimshaw et al. [37] investigated the tangential flow distortions induced by bleed ports, and their impact on the stability of the compressor. The distortion is the result of a complex interaction between velocity and pressure fields in the proximity to the bleed that alters the vector diagram seen by the downstream blade row. Such distortions are often the cause of anticipated stall and, to the knowledge of the authors, have never been studied by scale-resolved simulations.

Tip clearance. Axial compressors vanes and blades are often unshrouded because they need to be very thin to gain aerodynamic performance. The reduced blade cross-sectional area makes the profile unable to sustain the stresses associated with a shroud. Consequently, blades, and often vanes as well, are operated with a hub or tip clearance to avoid contact between stationary and rotating parts (see figure 3). The so-called tip clearance flow is often responsible for early stall, and may ignite surge if proper compressor operation corrective actions are not taken immediately. Such tip clearance flow is characterized by a strong shear layer that, departing from the pressure side, crosses the profile and reaches the suction side with a velocity vector strongly misaligned with the core flow. The resulting vortical structure is similar to a spiral, the intensity and size of which grows from leading to trailing edge, thereby provoking local blockage and spanwise flow rate redistribution. The accurate prediction of such flow features has been demonstrated to be of paramount importance to predict the onset of stall. You et al. [162] completed the LES of an axial compressor linear cascade with tip clearance. The results of their simulations were benchmarked with measurement with stationary and moving endwall. The results showed an excellent agreement with measurements in terms of tip leakage path and strength, together with the spanwise penetration of the secondary vorticity, a key aspect that impacts stall margin. The addition of casing treatment, as described by Juan et al. [52], can mitigate the negative impact on stall margin of the tip leakage flow, but it comes 
with additional aerodynamic complexity that simple RANS are not entirely able to capture.

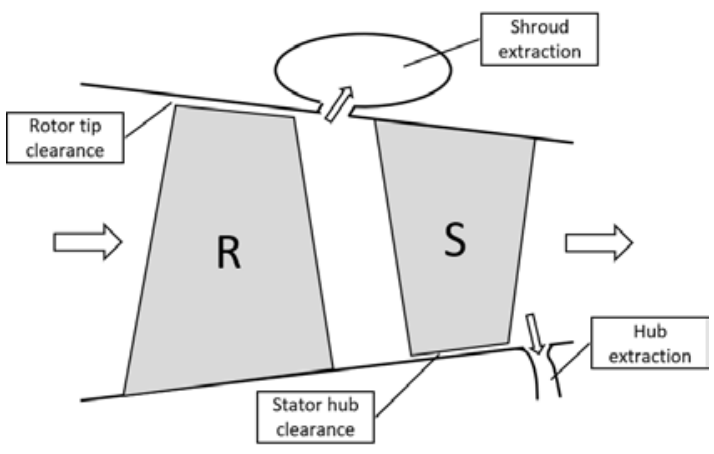

Fig. 3 Typical axial compressor sketch with unshrouded rotor $(\mathrm{R})$ and stator $(\mathrm{S})$ blades with hub and shroud extractions

Off-design. The compressor flow complexities described above are further exacerbated when moving to off-design conditions, when the compressor may be operated at the edge of stall, or even in presence of mild stalls on some stages that can be tolerated as long as the overall compressor characteristics remain stable. The flow field in such conditions becomes even more complicated due to the additional unsteadiness provoked by local separated areas and very thick wakes. In fact, when a compressor is operated strongly off-design, local stall phenomena, like multi-lobe rotating stall, may develop. Axial compressors may follow two different stall mechanisms. The first one is known as "modal stall", and it can be identified by the flattening of the pressure ratio curve moving from high to low flow rates. This is a clear indication of a gradual increase of aerodynamic blockage and losses. The second is the socalled "spyke-stall" that may happen when the pressure ratio is still growing while moving from high to low flow rate. In this case, while the compressor characteristics look healthy, the compressor develops a sudden stall that may quickly develop into surge. It is needless to say that the two different stall mechanisms require different cures. The difficulty here is that while modal stall can generally be predicted with moderate accuracy expectations by a steady calculation, spyke stall is essentially a compressor dynamic problem that requires multistage unsteady calculations. Stall was investigated by a dedicated experiment and RANS of a six stage axial compressor by Cornelius et al. [15]. Their simulation carefully maintained geometrical fidelity including fillets and clearances, and they ran steady mixing plane and non linear unsteady simulations completed by a systematic grid sensitivity study. Their results confirm the difficulties of RANS and URANS to predict the right flow capacity and stall point in a multistage compressor and highlighted how local separations grow at off-design conditions, where the predicted speedlines deviate the most from measurements. More recently, Yamada et al. [161] completed a DES of the flow in a multistage axial compressor. Their analysis revealed very complex flow structures growing from the interaction of hub clearance and corner stall. The simulations sug- 
gest that the accurate prediction of these flow features and the associated aerodynamic blockage is necessary to predict flow rate at which the compressor stalls. DES was still unable to predict the stall flow rate in agreement with experiments, although the mismatch was below $2 \%$. More worryingly, the numerical results also suggested that the predicted front stage pressure ratio was higher than in the experiments, while the opposite was true for the back stages.

The hub stall in axial compressors is difficult to predict. Scillitoe et al. [122] completed a detailed analysis of the three-dimensional separation in an axial flow compressor that accounted for the important effect of freestream turbulence intensity and endwall boundary layer state and demonstrated that the choice of the LES SGS model is critical by comparing with a companion DNS. The same authors [123] also investigated the effect of turbulence intensity and discrete incoming wakes on the suction side boundary layer state and its robustness to adverse pressure gradients. This information is essential in the design verification phase.

Deterministic unsteadiness. The axial compressor flow field is the result of the superimposition of a mean flow, a deterministic unsteady flow driven by adjacent blade row interaction, and a stochastic component, that is the turbulence field. While in turbines the deterministic unsteadiness can drive the generation of additional unsteady losses, in axial compressors the blade-row interaction, and the consequent potential effect and wake-profile interaction is known to have an impact on stall margin predictions. Henderson et al. [45] completed an extensive experimental campaign on a 1.5 stage axial compressor and took accurate measurements of the wakes and their impact on the stator row boundary layer with different levels of background turbulence. They also concluded that the correct prediction of wake dispersion is of paramount importance to the prediction of axial compressor performance. Unfortunately, Legget et al. [62] confirmed that RANS predicts wakes quite differently from LES. Zaki et al. [163] and Wissink et al. [157] both investigated the impact of incoming wakes on axial compressor blades by DNS. They selected the $V 103$ compressor blade for which measurements are available. In their investigations they concentrated on the midspan section assuming a homogeneous flow in the spanwise direction, as also done by Legget et al. [62] for the same test case. Zaki and Wissink both concentrated on wake dynamics and performance, with focus on the interaction of the incoming wakes with the boundary layer. Their fundamental investigations shed light on the boundary layer instability and by-pass transition triggered by the periodic disturbances carried by the incoming wakes. In particular Wissink et al. [157] studied the effect of the incoming wake strength on the boundary layer and concluded that strong wakes may keep boundary layers attached longer. This conclusion suggests that if wake decay is not predicted properly, like in RANS (see Legget et al. [62]) one could expect an incorrect prediction of the stall point. Axial compressor bladings are characterized by small thicknesses for aerodynamic performance, but this exposes the design to aeromechanics and flutter risks. In fact, thin profiles with limited camber may have a number of mechanical eigenfrequencies that must differ from aerodynamic excitation frequencies to avoid forced-response issues. The aerodynamic exitation is the outcome of the concerted action of pressure waves, wakes, and shocks in rotor-stator gaps. It is important to observe that the interaction between adjacent blade rows is driven by perturbations travelling both upstream and downstream due to 
the subsonic nature of the meridional flow. For variable speed machines and for any gas turbine that may be operated at part load, and during start-up and shutdown, it is virtually impossible to avoid the so-called "crossing", i.e. an aerodynamic forcing frequency that coincides with one of the mechanical natural frequencies. In this case, it is necessary to determine the actual blading response to the aerodynamic exitation, and what the associated mechanical stress is. In such circumstances, the exact prediction of the aerodynamic forcing in terms of frequencies, modal shapes, and phases, is critical. Thus, an incorrect aerodynamic forcing prediction will cause an incorrect mechanical stress prediction, and, once again, the accuracy of CFD is critical to success. Along these lines Espinal et al. [27] used a 1.5 stage high speed compressor to investigate the onset of non-synchronous vibrations by means of RANS. Their predicted frequency agreed fairly well with measurements, and also allowed to identify the unsteady flow mechanism responsible for the exitation.

URANS and LES. The short list of aero and aero-mechanics design challenges reveal that RANS or URANS are often unable to predict absolute performance, but they can often be of great help to determine trends and identify flow features. Hence, Joo et al. [51] investigated the rotor 37 tested at NASA by means of RANS and wallmodeled LES. While they could afford the prediction of a full speedline by RANS, the complexity of the flow field and the high Reynolds number $\left(1.3 \times 10^{6}\right)$ forced them to compute only one operating point with LES. Nevertheless, the scale resolved simulation predicted adiabatic efficiency, pressure and temperature ratio in much better agreement with measurements than the RANS. They also compared spanwise profiles of the same quantities and observed how LES was generally in better agreement with measurements, especially close to endwalls. They also noticed how LES was able to predict boundary layer transition that does affect the blade load distribution, at variance with RANS in which the boundary layer had to be tripped manually. Gourdain [34] completed a similar LES and URANS comparison with measurements on a different axial compressor stage. A dedicated grid sensitivity analysis showed how LES was very sensitive to resolution. The final grid was still fairly coarse, as witnessed by the fairly large size of the resolved flow structures. This is probably one of the reasons why LES showed a limited improvement over URANS in terms of speedlines or pressure ratio and efficiency. Nevertheless, the author compared the measured velocity profiles with predictions and found that LES was able to predict wake shapes much better than URANS. In particular, LES was capable of predicting the correct width and depth of wakes, a key factor to determine stator-rotor interaction and its impact on performance, aeromechanics, and acoustics.

McMullan and Page [76] used LES to accurately determine the performance of a controlled diffusion compressor blade profile at midspan. Their simulation proved the transitional nature of the boundary layer, despite the fairly high Reynolds number of 700,000 . They compared their predictions with measurements at design and off-design conditions observing how the loss prediction deviated from the expected value of strong off-design incidences. More recently, Legget et al. [62] performed a detailed comparison of LES and RANS with data of an axial compressor profile in a linear cascade with variable incidence. The experimental data set covered both design and off-design inlet angles and reported the so-called loss-bucket, key information to understand the operability of a compressor blade. The grid used for the 
LES was very fine and allowed resolution of around $98 \%$ of the turbulent kinetic energy, thereby reducing the error associated with the sub-grid-scale model (in this case the WALE model). RANS provided nearly as good a prediction as LES of profile load distribution, and of the loss-bucket. Some differences arose at off-design, and in particular at extreme negative incidence. As already observed in [34], the LES wakes were found to be quite different from RANS. This difference can be partly attributed to the different turbulent kinetic energy and velocity profiles in the vane, where LES showed larger reverse flow regions that impact the value of the trailing edge momentum thickness and finally the wake shape. By following the approach described by Denton [18], Legget et al. [62] split the losses into form or pressure loss, momentum loss, and blockage loss. This analysis was extended to the four incidence angles investigated, and revealed how the loss split differs between RANS and LES, although the overall loss level may be similar. More recently Laborderie et al. [57] completed a wall modeled LES of a three-stage axial compressor with IGV with the specific intent to investigate off-design performance and compare with measurements. They concluded, not unexpectedly, that sufficient grid resolution was critical in order for the LES to improve the match with measurements over URANS.

With the range of complexities described above, in conjunction with the latest axial compressor design trends towards the increase of pressure ratio and tip speed with and aggressive three-dimensional blading, CFD is becoming increasingly important as designs based on proprietary correlations are unable to describe the effect of detailed design choices and their impact on the flow. Gourdain et al. [36] conducted a comprehensive review of LES in industrial compressors and concluded that scale resolving simulation methods will not be practically applicable until 2035 due to the prohibitive mesh requirements in case all the geometrical and flow features described above need to be resolved. It is fair to conclude that, academia and industry are nevertheless both slowly moving in the direction of scale-resolved simulations of axial compressors, given the limiting inaccuracies of conventional CFD.

\subsection{Centrifugal Compressors}

Centrifugal v/s axial compressors. Centrifugal compressors find their applications in small aircraft engines and in industrial process compression. The reasons for their attractiveness is two-fold: firstly, small scale aircraft engines, with $<1 M W$ power, have very small axial compressor back stages that suffer of endwall and tip clearance effects that limit both their efficiency and operability range; secondly, the compression process takes advantage of centrifugal forces, and not only flow deflection, a physical process that is more robust to adverse pressure gradients and flow separation and, ultimately delays stall and widens operability [79]. Moreover, when very high operating pressures are necessary, above 300 bar, the centrifugal compressor guarantees a mechanical strength and reliability that an axial compressor is unable to give. The strengths of centrifugal compressors also represent the difficulties in computing the flow field, as the Reynolds number is generally very high due to the combination of high tip speeds and operating pressure, and the flow field is highly three-dimensional due to the concurrent action of the strong meridional curvature, as 
shown in figure 4, and blade-to-blade turning. RANS and URANS accuracy is therefore compromised by the strong secondary streamwise vorticity that interacts with the main flow, especially at off-design conditions where such interaction is amplified by the off-design loading of vanes and blades, as documented by Guleren et al. [38] who ran both RANS and LES of a low speed centrifugal compressor tested at NASA. They discovered that LES, unlike RANS, was able to predict separations and complex corner interactions that produced secondary vorticity in good accordance with measurements. An exhaustive list of publications on this subject can be found in Medic et al. [79].

LES applications. Despite the fairly high Reynolds number, often accompanied by transonic operating conditions, LES has been successfully used to investigate the flow in radial compressors, especially at off-design conditions where conventional RANS-based CFD fail as the flow develops large flow structures that interact with the main stream. In particular, Shahin et al. [125] used LES to compute the challenging near-surge operation of a radial compressor coupled with a vaned exit diffuser. The LES predictions compared well with data, and allowed to shed light on the complex mechanism associated with the full flow inversion across a surge cycle. These simulations allowed determination of the unsteady impeller load due to the significant flow dynamics associated with the surge event, and provide very accurate data for the mechanical design of the highly mechanically stressed rotating parts. Thus, on top of the added aerodynamic insight, radial machines benefit from LES for the mechanical design aspects as well. The near-surge aerodynamics were investigated by Hellstrom et al. [44]. Their analysis applied to a radial compressor coupled with an exducer typical of automotive applications in which the stall and surge of the compressor is generally dictated by a tip leading edge separation. LES proved able to predict the frequencies associated with the early stage of stall, while the detailed flow visualizations were helpful in following the stall mechanism in view of an improved redesign. More recently, the problem of a vaneless diffuser stall was investigated by LES of a simplified diffuser-like geometry that included the cavities around the impeller trailing edge [136]. In that case, LES shed some light on the development of reverse flow in the radial diffuser when running the compressor close to the left operating limit, revealing the close link that exists between the periodic perturbations from the impeller and the diffuser instability.

\subsection{High-pressure Turbine}

The aerothermal challenges of HPT. A detailed understanding of the aerothermal behavior of high-pressure turbines is another formidable challenge because the Reynolds numbers are high, transonic Mach numbers lead to shock waves that interact with boundary layers, and large scale flow and thermal variations generated in the combustion chamber are encountered at the inflow. Of particular interest is the interaction of the incoming unsteadiness with the HPT vane or blade boundary layers as it not only affects aerodynamic efficiency but also the heat transfer from the gas to the metal components and thus ultimately determines the life of the component. This is of paramount importance for both users and developers of GT as the residual life 


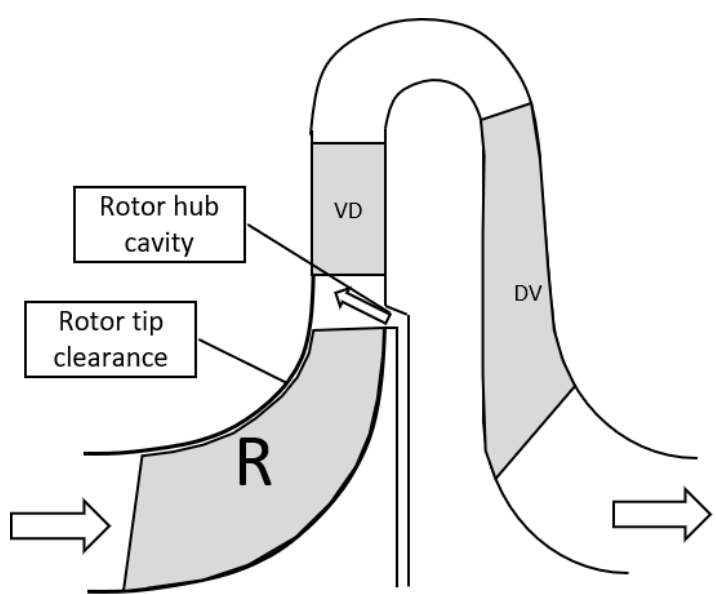

Fig. 4 Typical centrifugal compressor sketch with unshrouded rotor (R), vaned diffuser (VD), deswirler vanes (DV), and hub rotor cavity

and maintenance intervals are dictated by the so-called hot-gas-path that includes the combustor and the high-pressure turbine. As illustrated in figure 5, the stator is equipped with a hub labirinth seal while the rotor is unshrouded due to the combination of high tip speed and high gas temperature. The cavities need to be pressurized to avoid the risk of ingesting hot gas in regions of high mechanical stresses, like the disks that hold the rotor blades. The so-called purge air is extracted from the compressor (see figure 3) and it leaks into the main flow path thereby increasing the complexity of the endwall flow. All these details need to be captured properly by CFD.

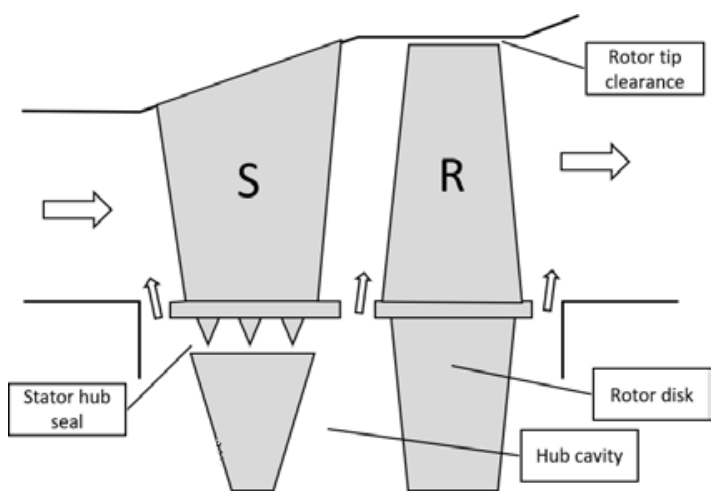

Fig. 5 Typical high-pressure turbine sketch with stator (S) equipped with hub seal, unshrouded rotor (R) and purged cavities

Cooling and performance impact. HPT design is driven by cooling requirements. Internal and external HPT cooling is a very sensitive subject as it is designed mostly 
by using industrial proprietary correlations that condense company multi-year experience. Therefore, GT manufacturers do not reveal their HPT cooling design details and the open literature only offers a large number of academic papers dealing with pre-competitive geometries and operating conditions. While in reality nearly all HPT vanes and blades, at least for the first stage, feature a range of internal and external cooling strategies, with film cooling significantly affecting the flow in the main gas path with the only exception of old generation heavy-duty gas turbines, studies conducted without cooling can enable designers to understand how much cooling air is actually required to achieve desired metal temperatures. Considering that a $1 \%$ cooling reduction may be worth more than $0.3 \%$ GT cycle efficiency, it is clear why such benchmarking is important as it allows to carefully balance cooling requirements with firing temperatures to reach the best overall cycle efficiency. Needless to say that any cooling flow reduction for better performance must be weighed against any potential life reduction. In addition, understanding the uncooled performance helps to quantify the impact of cooling on HPT aerothermal baseline performance. For example, Leach [60] measured the impact of coolant flow on aerodynamic efficiency of an HPT stator, quantifying that cooling resulted in an additional 3.5\% total pressure loss drop at 50\% span compared to a non-cooled stator. Along these lines, Sieverding et al. [126] and Michelassi et al. [85] investigated both experimentally and numerically the impact of HPT cooling ejection from both trailing edge and pressure side slots at various blowing ratios and isentropic Mach numbers. Their analysis revealed how trailing edge cooling ejection may have a positive impact on losses by changing the turbine base pressure, and also showed that RANS was unable to capture general trends with sufficient accuracy. While better overall predictive accuracy was achieved with URANS, this was not the case for the critical leading edge region.

Laboratory experiments that can record the turbulence properties within HPTs are very difficult due to the complexities of performing fast-response measurements within engine-scale experimental rigs, in particular for realistic operating condition such as high pressures and temperatures. It is the high operating temperature that may prevent optical access and may limit the applicability, accuracy, and life of temperature measurement devices. It is in this challenging scenario that high-fidelity simulations of HPT promise to provide the required information for future high-pressure turbines to be designed with increased aerodynamic performance and durability as well as to withstand even hotter gas flows with reduced cooling.

Scarcity of open literature measurements. The purpose of the comprehensive experimental campaign of Arts and Rouvroit [3] was in fact to provide a detailed data set useful to industry to determine the heat transfer coefficient of an uncooled HPT nozzle under a range of operating conditions in terms of Mach number, from subsonic to supersonic, and with realistic turbulent inlet conditions in terms of turbulence intensity. This data set, that dates back to 1990 is still extensively used for fundamental validation. Most of the high-fidelity simulations conducted to date have been guided by this experimental campaign that demonstrated the importance of free stream turbulence on the heat flux of an uncooled, transonic linear vane (LS89) cascade. The exit Reynolds and Mach numbers vary between $0.5 \times 10^{6}<R e<2 \times 10^{6}$ and $0.7-1.2$, respectively, at turbulence intensities from $1 \%$ to $6 \%$. It is worth mentioning that the variation of inlet turbulence intensity was achieved by changing the upstream 
position of the bar grid used to generate the turbulence. Although this is expected to also change the resulting turbulence length scale of the inlet condition, the resulting length scales were not reported. The impact of length scale is also very important, as shown in Pichler et al. [101], as the first HPT nozzle experiences the effect of large flow structures generated by swirlers in the combustion chamber and the consequent disrupting effect on the velocity and thermal boundary layers.

LES applications. One of the first high-fidelity simulations of a linear HPT cascade was conducted by Bhaskaran and Lele [6], who used LES to show that distortion of the turbulent inflow structures causes the formation of streamwise streaky structures on the leading-edge part of the vane and affects laminar turbulent transition further aft, with both effects significantly enhancing the heat transfer to the blade surfaces.

Ever since, a limited number of additional LES studies of HP turbine flows have been reported, with the majority focusing on evaluating the effect of inflow turbulence characteristics on the heat transfer to the vane surface. Considering the cases MUR129 and MUR235 of Arts and Rouvroit [3] for the LS89 geometry, Fransen et al. [29] showed that for high inlet turbulence intensity LES was able to correctly predict the aerodynamics and its impact on the heat transfer, while RANS failed in predicting the wall heat transfer. Subsequent LES of the same cases also revealed that the correct simulation of the incoming free-stream turbulence, and its decay, is crucial to determining the heat transfer on the blade surface [35]. It was found that near-wall streaks on the pressure-side greatly augment the surface heat transfer but tend not to lead to turbulent breakdown further downstream. The structures were attributed to the formation of Görtler vortices. However, Wheeler et al. [152] argued that the very low pressure-side curvature would suggest otherwise and showed that streaks are present on both the pressure-side and early suction-side, which have opposing curvatures.

Segui et al. [124] compared the properties of inflow turbulence generated either by separate pre-cursor simulations, as used in a number of previous studies $[6,50]$ and synthetic inflow turbulence considered by others (e.g. [101, 103]) and found no significant difference in heat flux predictions using either method. The sensitivity of the flow and heat transfer to inflow conditions including turbulence intensity and inlet/outlet pressure differences has also been investigated with LES [42]. The study concluded that the kinetic loss is mainly concentrated in the blade boundary layers and wake regions, at least in absence of discrete incoming wakes generated by an upstream blade row.

A series of highly resolved LES of the LS89 linear cascade were conducted varying the inlet turbulence intensity from $5 \%$ to $20 \%$ and the turbulence length scale between $5 \%$ and $20 \%$ of the axial chord [103]. It was found that both turbulent intensity and length scale play a significant role in the transition behavior and therefore the heat transfer to the blade.

To our knowledge the most (geometrically) advanced high-fidelity simulations to date are wall-modelled LES of an integrated combustor simulator/HPT stage configuration $[20,56]$. By comparing the results from the full coupled simulations with those from a standalone stage with the same mean inflow conditions and varying inlet turbulence, the aerodynamic performance of the turbine stage was found to be almost insensitive to the inlet turbulent conditions. However, the authors showed that 
realistic inflow conditions for the HPT, generated from an upstream combustor with the associated large scale flow structures generated by fuel/air injector swirlers, are critical to the temperature distribution and heat transfer.

First DNS applications. Wheeler et al. [152] performed the first DNS of a highpressure vane at transonic conditions with different levels of inlet turbulence intensity. They confirmed that at the leading edge and on the pressure surface near-wall streaks generated by stretching of the inflow vortices increased surface heat transfer but did not lead to transition to turbulence. On the suction side of the vane a strong interaction of pressure waves originating from the trailing-edge and the development of 2D Kelvin-Helmholtz-type instabilities were found to drive transition to turbulence, with the transition location varying significantly over time. The flow in the trailingedge region was found to be dominated by highly turbulent vortex shedding, with a broad turbulence spectrum. The DNS data allowed for a loss breakdown analysis that showed the time-mean strain, the turbulent dissipation and the irreversible heat transfer to contribute roughly $40 \%, 40 \%$, and $20 \%$ to the total irreversible entropy generation, respectively, with the majority of the turbulent dissipation generated in the near wake. Performing instability analysis using this DNS data, Zauner et al. [164] were able to predict with good accuracy the predominant frequencies of the turbulent structures found in the DNS close to the trailing edge. The linear stability analysis was also used to determine the most amplified spanwise wavenumber and thus provide a guideline for what spanwise domain size should be recommended for the scale resolving simulations focusing on midspan section.

Alternative numerical schemes. Several studies also assessed the ability of different solvers to accurately predict the flow and heat transfer on an HPT vane, comparing codes with structured and unstructured grids, implicit versus explicit solvers, and different numerical schemes. Unstructured grid capability is of importance due the geometrical complexity caused by the presence of film cooling holes and the need to include the blade interval cooling fluid feed cavities in the simulations. In Morata et al. [93] both structured and unstructured solvers captured the long stream-wise streaky structures responsible for the augmentation of the heat transfer and leading to the transition of the suction-side boundary layer. The study highlighted that unstructured LES, capable of modelling complex geometries, are able to capture the heat transfer accurately, although at higher computational cost than LES using structured grids. Pichler et al. [101] compared LES conducted with a structured high-resolution research code to results obtained with a commercial code on an unstructured grid for a statistically 2D linear transonic high pressure turbine vane cascade. In the unstructured LES the inflow turbulence was generated by explicitly meshing the turbulence generating grid while in the structured high-accuracy research code inlet turbulence was generated synthetically. The flow around the vane obtained from both codes was found to be in good agreement with reference data, however, differences were seen in the wake of the vane, i.e. the kinetic loss values were not the same. Bhaskaran et al. [5] also used different solvers with structured and unstructured grids and were the first to include the upstream bars for the inflow turbulence generation in a 6-th order accurate LES of the LS89 vane. The expected result was that heat transfer is controlled by the combination of turbulence intensity and length scale at the blade 
leading edge axial position, an information that is seldom available in the design phase, but that can have a large impact on performance.

Garai et al. [31] used a spectral element discontinuous Galerkin method with up to 16th order accuracy to conduct scale-resolving simulations of an HPT cascade, with focus on comparing natural transition occurring with clean inflow and bypass transition triggered by turbulent inflow. They reported that the turbulent characteristics of the vane boundary layers differed depending on whether the flow had undergone natural or bypass transition.

Noise. Another motivation for conducting high-fidelity scale resolving simulations is the study of the noise mechanisms, as prediction of aerodynamically generated sound requires accurate numerical schemes and resolution of a wide frequency range and the associated range of length scales. Comprehensive reviews on the numerical challenges of aeroacoustic simulations are available [13, 63, 137, 146]. Wang et al. [145] performed LES to study the noise sources in the fully three-dimensional transonic high-pressure turbine stage MT1. In particular the turbine noise generation mechanisms were studied for a steady inflow and one where a plane entropy wave train was injected leading to indirect noise generation, generated by the acceleration and distortion of entropy waves through the turbine stages. The indirect combustion noise was found to significantly affect the overall noise signature of the turbine. A subsequent study on indirect combustion noise [97] used Dynamic Mode Decomposition of the flow field and decomposed the noise field into upstream and downstream propagating waves.

Machine learning for HPT. Finally, high-fidelity scale resolving simulations of HPTs have most recently also been used to provide data for model development using data analysis and machine learning. In [116], LES of the LS89 cascade were performed to generate a data set covering different inlet turbulence intensities and inflow angles. Uncertainty quantification was then applied to the LES data to help build better predictive models. Weatheritt et al. [148] used the DNS data by Wheeler et al. [152] to develop nonlinear RANS closures using a symbolic regression approach based on gene-expression programming. A more detailed discussion of the translation of high-fidelity data to low-order models suitable for design will be given in section 7 .

The importance of the HPT on performance and durability of $G T$ is the main driver for additional applications of scale-resolving methods. The proven inability of conventional CFD to drive towards accurate and safe design is stimulating the investigations of simple cooling flows as a means to complete fundamental validation by both RANS and LES, or DNS, and constitutes the foundation for both a better understanding of the physics and for improving less computational intensive methods with the help of Machine Learning.

\subsection{Low-pressure Turbine}

\section{Low pressure turbine impact on GT performance.}

The low-pressure turbine (LPT) drives the propulsion device in aerospace applications, an electric generator in the case of power generation, or an external process 
compressor in the case of mechanical drive. While it generally operates in the highsubsonic regime, the LPT shares some of the aerodynamic challenges of the HPT, as illustrated in figure 6 , due to the presence of seals and cavities that need to purged to avoid hot gas ingestion. The lower gas temperature together with the lower tip speed allows to equip the rotor blades with shrouds. Unshrouded HPT rotors generally evolve along axial endwalls to control the tip clearance, whereas LPT rotors are generally shrouded thanks to the reduced rotational speed and lower gas temperature. Both hub and shroud walls may be inclined with respect to the axial direction (see figure 6) to expand the flow along the span, reduce the exit Mach number and the associated kinetic losses together with jet noise.

The performance of the LPT directly affects the efficiency of the entire gas turbine and thus is of great importance. The operating conditions of an LPT in this wide range of applications change in terms of Mach numbers, from low to high subsonic, and Reynolds numbers. The Reynolds number needs special attention as in propulsion applications it may range from 30,000 to 300,000 depending on the engine size and the altitude of operation, from take-off to cruise. Such a small number is driven by the small profile chord, to reduce weight and have boundary layers as laminar as possible, the low revolution speed dictated by the speed of the propulsion fan, at least in direct drive applications, and the low operating pressure at altitude. Conversely, for ground based applications, like power generation of mechanical drive, the Reynolds number is generally higher around $1 \times 10^{6}$.

In summary, the small chord-size of both vanes and blades and their operation in low air density environments, the aero-propulsion LPT generally experiences significantly lower Mach and Reynolds numbers than the HPT or the HP compressor and therefore is more amenable to high fidelity simulations. At the same time, this combination of low Mach and Reynolds number, in conjunction with the very high loading of modern designs, in an effort to reduce engine weight, size and cost, results in the suction and pressure side boundary layers becoming prone to laminar separation and subsequent laminar-turbulent transition of the separated shear layers $[39,75]$.

Separation bubbles and the type and location of laminar-turbulent transition are sensitive to upstream disturbances, including background turbulence in the main stream and wakes from the upstream rows $[26,46]$. Such flow separations, happening mostly on the suction side after peak suction, are to be avoided at design conditions as they obviously constitute an excessive source of losses, but they can be tolerated in off-design conditions provided the separation bubble reattaches before the blade trailing edge. In such a case, the profile loss growth, that is proportional to the blade trailing edge suction side momentum thickness, is acceptable.

Therefore, as the profile losses depend strongly on the state of the boundary layers on the blades [43], it is crucial that the boundary layer states, and the presence of a local boundary layer separation, can be predicted accurately in order for LPT design to match stringent performance and weight requirements. The above characteristics pose considerable challenges for turbulence modelling approaches that need to specifically address boundary layer transition of the by-pass and separated flow type, while this class of internal flows do not experience natural boundary layer transition due to the concerted action of high background turbulence and discrete wakes and pressure waves. 
Although Reynolds Averaged Navier-Stokes (RANS) incorporating the latest transition models appear to achieve good agreement with experiments at low Reynolds numbers in terms of pressure coefficient and transition onset, these models are often unable to provide accurate results in terms of total pressure losses $[54,96]$ in the wide design space generally required for changing operating conditions of LPT. Also RANS are often found inadequate to resolve the complex interactions of periodic inflow perturbations, often combined with large background turbulence intensity, with the boundary layers. For these reasons, the LPT was one of the first gas turbine components to be investigated with high-fidelity simulations. As the LPT has possibly received more attention with DNS and LES than the other gas turbine components reviewed in this contribution, the following overview is far from complete and summarizes a number of milestones that admittedly will be subject to the author's inevitable bias.

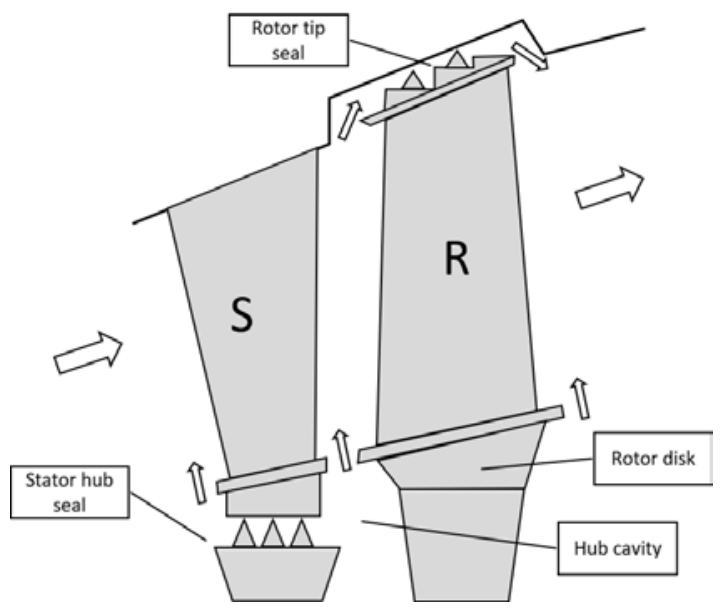

Fig. 6 Typical low-pressure turbine sketch with stator (S) equipped with hub seal and shrouded rotor (R) with purged cavities

Early DNS and LES. The first incompressible flow DNS of a turbine cascade was performed by Wu \& Durbin [158] who observed a Kelvin-Helmholtz-type roll up mechanism on the aft section of the suction side and described longitudinal vortical structures forming on the pressure side induced by the incoming wakes for all phases. Additional incompressible DNS have contributed further to the understanding of the effect of incoming wakes on the pressure and suction side boundary layers $[89,154,156]$, in particular how these incoming wakes affect the suction side separation bubble and consequently the profile losses. Despite the low speed of LPTs, the flow speed is high subsonic and at peak suction the local isentropic Mach number can reach values of 0.6 or more. Under these operating conditions, a simulation performed under the assumption of incompressible fluid will overestimate the peak Mach number, and will locate it upstream of where a compressible simulation will indicate, as proven by Michelassi et al. [89] who compared incompressible DNS and 
LES with compressible URANS. Therefore incompressible simulations will overpredict the strength and extent of the adverse pressure gradient region on the blade suction side after peak suction, and will probably overestimate the losses in this critical flow region.

Highly loaded LPT. Several early studies also focused on flow control for loss reduction, considering low Reynolds number cases with pronounced separation bubbles. Numerical simulations were conducted of highly loaded LPT blades at low Reynolds number $(25,000)$, assessing the benefits of pulsed injection vortex control on losses [112]. By mitigating the suction side separation, wake losses were shown to be reduced by as much as $22 \%$. Inspired by the success of the experimental study by Sondergard et al. [130] where vortex-generators jets were used to control separation on the suction side of an LPT, Postl et al.[108] used DNS to investigate the potential of pulsed vortex generator jets to control laminar separation. However, this study was conducted for a setup using a flat plate, with suitable boundary conditions to produce a laminar separation bubble. It was shown that pulsed vortex generator jets were more effective than their steady counterparts for the same momentum coefficient used for the actuation. Although such fundamental studies are indeed of interest, the question on the robustness to fouling of such devices remains and despite the proof-of-concept completed by scale-resolved simulations, they are not implemented in real engines yet.

High-fidelity simulations at realistic operating conditions. To reduce computational cost, large eddy simulation were performed of LPT turbine cascades in a number of studies [74, 111, 119]. Matsuura \& Kato [74] performed compressible flow LES of an LPT cascade at Reynolds numbers 500,000 with no and 5\% freestream turbulence levels and noticed that without inflow perturbations pressure waves propagating from the blades wake have a noticeable effect on the separated-boundary layer near the trailing edge. A similar phenomenon was also observed by Wheeler et al. [152] on an HPT. Medic \& Sharma [78] performed compressible flow LES using the WALE subgrid-scale stress model of three different LPT midspan sections for a Reynolds number range of 30,000 to 150,000 . The freestream turbulence intensity was varied and detailed comparisons with experimental data showed that the LES captured the main trends across all geometries quite well as opposed to RANS. However, the LES suffered from difficulties in predicting boundary layer transition and capturing the delay of laminar separation with varying inlet turbulence levels. Such difficulty of LES was also observed and documented in [88] where LES predicted a delayed by-pass transition with respect to DNS.

In a large-eddy simulation study of the T106D-EIZ linear cascade at Reynolds number 60,154 the effects of inlet freestream turbulence intensity on aerodynamic performance as well as temporal and spatial evolution of coherent structures in separated shear layers were investigated [165]. The incoming freestream turbulence levels varied from 0 to $10 \%$. The results showed that the open separation present on the suction side could not be removed even by the highest inlet turbulence levels, but nevertheless could be significantly reduced in size. It was shown that the coherent structures in the separated shear layer were significantly affected by the inflow conditions. 
DNS of a linear T106A LPT cascade at isentropic exit Reynolds number 51,831 without incoming wakes or freestream turbulence were recently conducted with a finite volume code [110]. It was found that cell counts previously considered to be high for such a Reynolds number, e.g. 95 million points, could produce qualitatively different results to an even finer mesh (161 million), such as a leading edge separation and no laminar separation upstream of the trailing edge.

Hammer et al. [40] performed well-resolved LES of a linear T106A cascade in which the upstream wake-generating bars were either only translated in the pitchwise direction or rotated clockwise or anti-clockwise while moving, in order to generate different wake shapes and turbulence characteristics. The results indicated that in all cases the wakes triggered boundary layer transition and thus intermittently prevented separation on the suction surface. Of particular interest was the observation that the weakest wakes led to the largest profile losses, due to their inability to fully suppress the separation bubble, but resulted in the smallest overall LPT loss, presumably as they experienced the smallest wake distortion loss.

Design of experiments with high-fidelity simulations. To address some of those shortcomings, and benefiting from the increased available computing power, Sandberg et al. [117] performed the first compressible flow DNS of the linear T106A LPT cascade. It was found that for compressible flow simulations acoustic waves generated at the blade trailing edge could accumulate in the domain if the in- and outflow boundary conditions were not successfully preventing spurious reflections, and thus could lead to an (artificial) in flow turbulence intensity of close to $1 \%$. More importantly, it was found that when comparing to presumably 'clean' experimental data, it was important to specify the same inflow turbulence intensity, even for intensities well below $1 \%$ in order to correctly predict the kinetic wake loss profiles. With the aim of supporting and guiding the LPT design process, a series of DNS were then conducted with incoming wakes at various reduced frequencies [86], different inflow turbulence intensity levels and for two Reynolds numbers, 60,000 and 100,000 , with a total of 12 cases. A detailed loss generation analysis revealed that unsteady losses caused by the incoming wakes experiencing significant distortion in the passage significantly contributed to the overall losses for reduced frequencies for which the wakes remained distinct and did not mix with each other before entering the variable area section. The study also interrogated the DNS data to examine the validity of the Boussinesq eddy viscosity assumption, and showed that the error made by the linear stress-strain relationship used in many RANS models was highest in the trailing edge region. At the same time, it was found that the minimum achievable error, or a so-called entitlement, for a linear RANS model was about a factor of 3 to 4 smaller than the error computed using a standard $k-\varepsilon$ model applied to the DNS data. This set of DNS constitutes the first attempt to complete a design-of-experiment based on scale-resolving CFD and it allowed to investigate a design space of specific industrial interest. Moreover, the DNS postprocess in [86] attempted to use the data as a virtual test by applying the control volume approach based on the theory illustrated by Denton [18]. Doing so it was possible for the first time to use DNS to dissect different source of losses generally identified by LPT aero-designers, and quantify the additional losses caused by the unsteady interaction of the profile with incoming wakes. The simulations proved that the variable pressure incoming wake 
mixing that takes place between the turbine leading and trailing edges is responsible for the unsteady losses that are an amplification of constant pressure mixing of the same incoming wakes. LES has been also used to investigate the concerted impact of several important design parameters, like the revolution speed, the through-flow velocity, the vane and blade count. These numbers can be condensed in the so-called reduced frequency and flow coefficient, that measure the unsteadiness generated by incoming wakes seen by the downstream blade row and the ratio of the through flow velocity with the revolution speed respectively. While it is well known that these two parameters alone are worth a considerable portion of the lost efficiency, scaleresolved simulations have never been used before to complete the investigation of the associated design space. The LES results of [84] suggested the existence of a best combination of these two parameters, the impact of which was condensed in performance trends. Quite interestingly, the simulations revealed that the unsteady losses were proportional to the normal distance between the incoming wakes, suggesting that when wakes interact and merge before the blade leading edge the expected unsteady losses are small. Moreover, the simulations indicated how the unsteady load pattern changes on the blade, as the largest unsteadiness moved in different chordwise positions with potential impact on the aeromechanics and flutter response. It is also important to remind that the LES results were anchored to DNS to make sure the subgrid scale model did not introduce excessive bias to the results.

Moving closer to engine configuration. It is common practice to investigate the impact of discrete incoming wakes on LPT blades by a set of upstream moving bars. So doing the bars can de designed to produce realistic wakes, but such approach is unable to reproduce the potential effect that takes place in the axial gap between a stator and rotor blade of a turbine. To overcome such limitation the only alternative is to perform a complete stage simulation, as done by Pichler et al. [102] who completed highly resolved LES of the blade-to-blade interactions in a realistic LPT stage. The simulations were conducted at exit Reynolds number 100,000 and $M=0.6$ for modern blade sections. To determine the performance derivative, the gap size between stator and rotor was varied from $21.5 \%$ to $43 \%$ rotor chord. A loss breakdown analysis allowed to identify the prevailing loss mechanisms as a function of the gap size. It was found that in the large gap size case, the turbulence kinetic energy levels of the stator wake close to the rotor leading edge were only one third of those in the small gap case, due to the longer distance of constant area mixing. The small time averaged suction side separation on the blade, found in the large gap case, disappeared in the small gap calculations, confirming how stronger wakes can keep the boundary layer attached. The higher intensity wake impinging on the blade, however, did not affect the time averaged losses calculated using the control volume approach of Denton. On the other hand, losses computed by taking cross sections upstream and downstream of the blade revealed a greater distortion loss generated by the stator wakes in the small gap case. Despite the suction side separation suppression, the small gap case gave higher losses overall due to the incoming wake turbulent kinetic energy amplification along the blade passage.

The majority of high fidelity simulations addressed the midspan section, as this is the portion of the flow where the majority of losses are generated. Recently the endwall region is gaining attention as, while its impact at the single blade row level 
is not as important as the midspan section, it controls the majority of the losses carryover to the downstream rows.

As an example, the study by Cui and Tucker [16] addresses endwall flow and the interaction with the cavity purge flow by LES. Although in LPT endwalls are not as important as in HPTs, their impact on performance is quite significant, especially for the front stages of an aircraft engine multistage LPT.

Recently high-resolution LES have been conducted of a linear low-pressure turbine cascade including endwalls [106] at an exit Reynolds number of 120,000. The authors showed results for two different grid resolutions and concluded that there were still subtle changes in the endwall vortex system prediction between the coarser and finer mesh and thus recommended that the finer mesh resolution is necessary. The study was conducted using incoming endwall boundary layers with different momentum thicknesses and the endwall vortex characteristics and the associated loss were shown to be considerably affected. The results further revealed that RANS calculations, if performed following strict best practice rules, agreed well with LES, in particular in the prediction of the end wall vortex system, with the only significant differences observed in the loss generation of the blade wake, due to the inability of correctly predicting wake mixing [73]. Ciorciari et al. [11] performed a similar investigation in presence of discrete incoming wakes generated with moving bars by running both a set of linear cascade experiments and the companion CFD simulations. The results suggested that the endwall region is moderately affected by the deterministic unsteadiness provoked by the upstream wakes.

With respect to the aerodynamic load of the T106 blade, the geometry predominantly used in high-fidelity simulations, latest design trends indicate a load increase and quite different pressure distributions, as witnessed by the geometries investigated by Medic \& Sharma [78]. Consequently, the Zweifel number, that is a measure of the aerodynamic load, is growing in the attempt to reduce blade and vane count, product cost and weight, without jeopardizing performance. As a result the suction side boundary layer is longer exposed to increasingly large adverse pressure gradients. At the same time, chord Reynolds numbers are moderate as a consequence of small chord-size of the blades and their operation in low air density environments, like at cruise conditions. This combination results in the suction and pressure side boundary layers becoming prone to laminar separation and subsequent laminar-turbulent transition of the separated shear layers $[39,75]$. Separation bubbles and the type and location of laminar-turbulent transition are sensitive to upstream disturbances, including background turbulence in the main stream and wakes from the upstream rows $[26,46]$. As the profile losses depend strongly on the state of the boundary layers on the blades [43], it is crucial that the boundary layer states can be predicted accurately in order for LPT design to match stringent performance and weight requirements. Although Reynolds Averaged Navier-Stokes (RANS) incorporating the latest transition models appear to achieve good agreement with experiments at low Reynolds numbers in terms of pressure coefficient and transition onset, these models are often unable to provide accurate results in terms of total pressure losses [54] in the wide design space generally required for changing operating conditions of LPT. Also RANS are often found inadequate to resolve the complex interactions of periodic inflow perturbations, often combined with large background turbulence intensity, with 
the boundary layers. The design trends and the risk associated with RANS are the main reasons why LES of LPT is gaining momentum [74, 111, 119], owing to the improved accuracy achieved by resolving the energy-containing structures in the flow while keeping computational cost at bay by modeling the small scales of turbulence. However, as mentioned before, LES suffers from difficulties in predicting boundary layer transition and capturing the delay of laminar separation with varying inlet turbulence levels [78], or due to periodic incoming wakes [88].

The concerted action of incoming turbulence intensity and surface roughness does impact the suction side boundary layer and may promote earlier reattachment in the presence of an adverse pressure gradient induced separation, as indicated by the very fundamental studies of Nagabhushana et al. [94]. In another LES study [41], highresolution LES was used to conduct numerical experiments of the effect of an as-cast roughness patch on the suction side of a T106A LPT to investigate their effect on losses. However, maybe more importantly they also demonstrated that a roughness model, with the required parameters chosen appropriately, could reproduce the fully represented roughness results, both in terms of transition behaviour on the blade and kinetic wake loss in the wake.

Alternative numerical schemes. Over the last few years, the suitability of discontinuous Galerkin and spectral-element codes for DNS of low-pressure turbine cascade simulations has been assessed. Garai et al. [30] performed DNS of a linear T106D cascade and reported that the method was able to capture the main features of the flow, and attributed subtle differences between DNS and the reference data to the fact that freestream turbulence was not included in the simulation. In the DNS of the T106A cascade by Cassinelli et al. [9], again no inflow turbulence or wakes were included. The effect of the polynomial order used, the spanwise domain size and the spanwise resolution were investigated and results were compared with experimental data up to exit Reynolds number 160,000, showing excellent agreement. High-order Flux Reconstruction schemes have also recently started to see deployment for turbomachinery applications. Vincent et al. [143] performed simulations of a linear LPT cascade primarily to demonstrate the performance of the code, reaching up to $58 \%$ of peak system performance on unstructured grids and sustained computation up to 13.7 double precision PetaFLOP/s. To assess the suitability of flux-reconstructions schemes using strongly stretched and warped grids, as commonly encountered in turbomachinery applications, Trojak et al.[139] performed simulations of several canonical test cases and found good wave propagating capabilities.

\section{Alternative postprocessing}

Alternative postprocessing: POD. The DNS and LES data sets are extremely rich and contain a large amount of important information that is not trivial to extract. This is particularly true for cases that feature both stochastic and deterministic unsteadiness, as found, for example, for stator-rotor interaction. A question that often arises is how much is unsteadiness worth in terms of losses, and what are the respective unsteady flow features responsible for loss generation. The direct analysis of computational results can provide answers to the first half of the question, while the second 
half requires more work. Proper Orthogonal Decomposition $(P O D)$ has emerged as a useful method to analyze measurements and it was successfully applied to $L P T$ linear cascade data by Lengani et al. [65], who discerned flow features directly and not directly related to the passing wakes. The same method was also successfully applied to LES datasets [64]. The LES data provided a detailed set of flow statistics that allowed the direct computation of the entropy rate of change equation. Crucially, Lengani et al. [64] managed to project some of the entropy equation terms onto each $P O D$ mode, making it possible to associate the contribution of each mode to loss generation. Considering that POD is able to discern deterministic from stochastic unsteadiness, as elaborated in Lengani et al. [64], it is then possible to rank each unsteady mode (or groups of modes), related to certain physical mechanisms, in terms of how much loss is generated, and this could guide both the development of turbulence models as well as the aerothermal design of $L P T$ by pointing to the top-offenders.

Alternative postprocessing: Entropy Loss Analysis. An entropy-based simulation quality framework was also recently developed to assess the quality of RANS calculations [168]. The entropy transport equations based on averaged flow quantities were derived, and the entropy generation process decomposed into the salient physical mechanisms, such as mean viscous dissipation, turbulence production, mean and turbulent heat flux, etc. Applying the framework to LES and RANS results of a VKI LS-89 HPT vane, it was shown that entropy generation caused by mean flow features were in close agreement between LES and RANS, suggesting good overall RANS mean flow predictions. However, the turbulence production contribution to loss in the vane wake was not captured well by RANS, constituting the main reason for an inaccurate total loss prediction by RANS.

\section{Ensure high-fidelity CFD quality}

This section is motivated by the authors' observation that past studies have used the term 'DNS' and 'LES' rather loosely. Claiming that a simulation is a DNS because no modelling was invoked is clearly not appropriate. However, how do we decide when to call a simulation DNS or LES? In the purest sense, a true DNS is a simulation in which all length- and time scales are fully resolved by the numerical approximation. In the context of turbomachinery simulations, many studies have settled on assessing the grid quality based on first-order statistics, such as mean pressure distribution on the blade and wake losses. In contrast, DNS studies of more canonical flows, turbulence quantities like turbulence kinetic energy or its dissipation and their spectra have been assessed in addition to mean quantities to show grid independence (see, e.g., Lee and Moser [61]). There is no good reason for why these more stringent criteria should not also apply to turbomachinery flows.

In the following, a case study is provided to establish a relationship between required grid resolution and certain quantities of interest. High-fidelity simulations of a linear low-pressure turbine cascade are chosen as the moderate Reynolds number does allow for a rigorous grid independence study with current computational resources. However, it is the authors' belief that most of the conclusions made can be 
extrapolated to cascade simulations at higher Reynolds numbers. For general grid count estimates for other turbomachinery applications, see section 6 in Tyacke and Tucker [141]. First, a brief review of grid-resolutions used in selected previous studies is given, followed by a discussion on the requirements of the various characteristic flow regions present in an LPT cascade simulation. Then LPT simulations using one particular high-fidelity compressible flow solver on a range of different grids are presented and used to investigate the grid quality needed to achieve grid convergence for quantities related to either the mean flow, the energy-containing large turbulence scales or the energy-dissipating small turbulence scales.

Along these lines, industrial companies generally determine the most appropriate grid structure and resolution in combination with boundary conditions in the form of CFD best practices to minimize the risks of getting inaccurate design trends. While RANS and URANS are still the workhorse of the CFD assisted design, the open literature suggests turbomachinery companies are looking more and more into LES the accurate execution of which will require the development of best practices for scale resolving simulations.

\subsection{Grid resolution used in previous high-fidelity simulations of LPTs}

We start by summarizing grid resolutions that have been used in select previous studies in table 1. It is of course difficult to directly compare the resolutions used in these studies due to the complex interdependencies of grid resolution and numerical schemes. Thus the different cases are listed here mainly to highlight that very different resolutions have been considered sufficient in different studies.

\begin{tabular}{l|c|c|c|c|c|c} 
Ref & $R e_{i s}$ & spatial scheme & $n_{p}$ & $n_{s p}$ in thousands & $n_{\text {tot }}$ in millions & $x^{+}, y^{+}, z^{+}$ \\
\hline$[159]$ & - & $\mathrm{FV}$ & 410 & 128 & 52 & $24,-, 11$ \\
{$[158]$} & $148,000^{*}$ & $\mathrm{FV}$ & 440 & 129 & 57 & - \\
{$[154]$} & $60,000^{*}$ & $2^{\text {nd }} \mathrm{FV}$ & 263 & 64 & 16 & - \\
{$[53]$} & $148,000^{*}$ & $\mathrm{FV}$ & 665 & 129 & 57 & $28,2.3,19$ \\
{$[156]$} & 100,000 & $2^{\text {nd }} \mathrm{FV}$ & - & - & 93 & $25,1.7,15$ \\
{$[105]$} & 60,000 & $4^{t h} \mathrm{FD}$ & 993 & 98 & 132 & $8,1.0,6.5$
\end{tabular}

Table 1 Simulation details driving accuracy and cost of published LPT DNS where $R e_{i s}, n_{p}, n_{s p}, n_{t o t}$ are the isentropic exit Reynolds number, the number of points in the blade to blade plane, the points in the spanwise homogeneous direction, and the total number of points. The last column shows the streamwise, wall normal and spanwise spacings in wall units. FV denotes finite volume methods, FD denotes finite difference, with preceding numbers indicating the formal order of accuracy.

To study the interaction of incoming wakes with the suction side boundary layer of an LPT blade, Wu and Durbin [159] simulated a flat plate boundary layer subjected to an LPT-inspired streamwise pressure profile. The grid design was consistent with previous flat-plate boundary layer studies in that the first 10 grid lines in the wallnormal direction were located below a $y^{+}$of 9 . To present grid convergence they investigated the incoming wake profiles of the precursor simulation. For the actual flat plate simulations the mean velocity profile and the Reynolds stress components 
across the boundary layer were presented. Wu et al. [158] subsequently used the grid quality established in their previous flat-plate simulations for real turbine geometry simulations and showed that the mean pressure distribution around the blade agreed well with experiments [134]. Kalitzin et al. [53] also focused on adequately resolving the blade boundary layer, further decreasing wall spacing at the expense of coarsening the passage with respect to the study by Wu et al. [158]. Wissink [154] and Michelassi et al. [88] used the mean pressure distribution and wake loss to demonstrate grid convergence and Wissink and Rodi [156] used the blade pressure distribution to conclude on grid convergence. Over the next ten years most of the subsequent DNS investigations of LPT flows have used similar grid resolutions to the above initial DNS studies. We do not list all of them here for conciseness. To the authors' best knowledge, only Pichler et al. [105] also reported detailed turbulence quantities, such as turbulence dissipation rate, for their LPT simulations and used a significantly finer grid than previous studies considering an isentropic exit Reynolds number of 60,000.

As for LES studies, Michelassi et al. [88] compared their data to DNS results and showed good agreement in terms of pressure distribution and mean wake loss, suggesting that LES are capable of accurately predicting the mean flow for the same blade as used in many of the aforementioned DNS studies at considerably reduced resolution. Still, LES predicted a delayed suction side boundary transition with respect to DNS. As an additional example, Medic and Sharma [78] conducted a series of LES studies at different Reynolds numbers and found significant differences to benchmark measurements in particular for low Reynolds numbers, presumably as the grid did not support transition to be accurately captured.

\subsection{Grid-requirements in different regions}

Unlike canonical flow cases such as channel flow, where grid resolution requirements can be established in a straight forward manner, the needed resolution in the different flow regions present in cascades might be driven by different requirements, especially in presence of incoming wakes. The required resolution in each region is determined by the smallest scales that have an effect on the quantities of interest. This can mean that the smallest scales needed to be resolved are those that directly affect the turbulence kinetic energy, or those that only have an indirect impact on the mean flow, for example by leading to delayed transition prediction on the suction side of an LPT blade. The effect of insufficient grid resolution on the simulation results depends on the numerical scheme used. For high-accuracy solvers with little numerical dissipation, not resolving the smallest scales may lead to an overprediction of energy in the scales larger than the grid cut-off. However, this might not manifest itself in quantities that are mostly determined by the large-scale behavior, such as the mean flow or level of turbulence kinetic energy. When using codes with low-order accuracy the aliasing of energy from the unresolved scales to resolved scales might not lead to any overprediction of energy in the inertial subrange as the excess energy is dissipated by the numerical method. Nevertheless, it is the authors' view that regardless of what numerical method is employed, a substantial amount of the inertial sub-range needs to be resolved such that there is no significant influence on the large scales driving 
mean flow quantities and first order statistics. Should the grid resolution become too coarse, i.e. too small a part of the inertial subrange is resolved, the largest scales will be affected by resolution and even mean flow quantities will be compromised.

In the following we divide an LPT flow into three characteristic regions in which different scales dominate, namely the free-stream, the blade walls and the wake region. For each of these regions, we can make comparisons with canonical cases for which a wealth of information on how to establish grid independence is available.

Upstream of blade. In this region, turbulent fluctuations are due to background turbulence and/or incoming wakes. In the case of compressible flow simulations, additionally perturbations induced by aerodynamic noise generation can also be present and without special treatment can contribute to a disturbance level of $0.5 \%$ that might affect suction side transition [117]. In a turbomachinery environment, it is unlikely that the incoming turbulence upstream of a blade can be classified as isotropic decaying turbulence, since the disturbances are comprised of discrete wakes of blade rows and other flow devices upstream. In experiments background turbulence is mostly generated using an upstream grid and the turbulence approaching the blade in the constant area section can be classified as decaying turbulence, which has been the subject of many studies (e.g. [14]). However, to model the influence of wakes of upstream rows many experiments have employed cylinder bars upstream of the blade row (also referred to as wake generators) that move in the pitchwise direction (e.g. Liu and Rodi [70] and Schulte and Hodson [121]). The wakes shed by these bars are essentially cylinder wakes and spectra measured in these wakes will show differences to the theoretical turbulence cascade at the large scales while showing a similar inertial subrange and dissipation range at the small scales (e.g. Braza et al. [8]). Hence, in the latter case of incoming wakes the resolution requirements might be less stringent than in the case of incoming decaying turbulence in order to obtain the correct flow field interacting with the blade because it might be sufficient to resolve the dynamically relevant larger scales. Comparing the incoming turbulent flow from high-fidelity simulations to engine data or experiments is generally very difficult because detailed information on its characteristics, such as anisotropy, turbulent length scales or frequency spectra are not available. Nevertheless, in the simulations an inlet condition needs to be specified and the streamwise evolution of that inflow condition can be assessed to determine whether the grid resolution is sufficient.

Blade boundary layers. Here the interaction of the flow with a wall drives the solution. Initially, laminar boundary layers form from the front stagnation point that are subjected to continuous (free-stream turbulence) or/and intermittent (upstream wakes) disturbances and the grid resolution has to be sufficiently high to be able to correctly represent the physical receptivity process and to resolve the subsequent evolution of the boundary layer disturbances that can affect the laminar-turbulent transition. Laurent et al. [59], who simulated a flat plate with a prescribed adverse pressure gradient subject to upstream disturbances, recommended that the required resolution in this area be comparable, if not more stringent, than in a turbulent boundary layer directly after transition. 
For wall bounded flows the situation is different because even the smallest (nearwall) scales are of crucial importance. In transitional regions of the flow insufficient resolution might affect the level of forcing in the receptivity region (see, e.g., Mayle [75]) and might also damp disturbances inside the boundary layer. Either aspect results in a delayed transition or an earlier separation, depending on the blade profile and Reynolds number and, thus can have a significant impact on the global flow behavior. To complicate things, the near-wall LPT flows in the typically considered Reynolds number range are hardly fully turbulent, e.g. in simulations at isentropic exit Reynolds numbers of 60,000 where incoming bar wakes promote transition, the momentum thickness Reynolds numbers, defined as

$$
\operatorname{Re}_{\theta}=\frac{\rho_{i s, \text { exit }} V_{i s, e x i t} \theta}{\mu\left(T_{i s, \text { exit }}\right)},
$$

is approximately 360 [86]. This is a low value, in particular compared with previous DNS studies that have focused on just the boundary layer subject to an adverse pressure region [12]. Hence, this might partly explain why some LPT simulations performed to date, as listed in table 1 , have used coarser wall resolutions than pure boundary layer or channel studies, where values of $x^{+}=5-10, y^{+}<0.5$ and $z^{+}<6$ are common [61].

Blade wake. Similarity to the upstream incoming flow can be assumed, particularly once the wake has fully developed and a turbulence cascade, as occurring for an equilibrium turbulent flow at sufficiently high Reynolds number, is recovered [7]. In the near wake, for thick trailing edges often vortex shedding occurs and the shear layer velocity gradient will lead to significant turbulence production and a subsequent redistribution of turbulence kinetic energy in the wake. These mechanisms are predominantly large-scale structure phenomena for which grid requirements are more benign. However, the near-wake region is fed by flow coming off the trailing edge of the blade and thus the grid requirements depend on the characteristics of the aft boundary layers. If the boundary layers are turbulent, the grid needs to support resolution of the streamwise aligned structures, known as streaks. The near-wall turbulent boundary layer structures lose this streamwise orientation just upstream of the trailing edge to become mainly spanwise orientated in the near wake region [118]. This has implications on the grid design but in most cases is inherently addressed by the typically significantly tangentially refined grids in the trailing region.

A detailed grid resolution study for a linear LPT cascade at isentropic Reynolds number of 60,000 was recently conducted, assessing the sensitivity of different quantities to varying grid quality in the three regions discussed above [104]. A summary of the setup and the key findings is provided in the following, while for more details on the grid properties, such as the blockwise grid counts and orthogonality, and statistical convergence of the results the reader is referred to the original paper. However, for completeness, Figure 7 shows the 9-block topology employed for the coarsest DNS conducted of the LPT case. An O-type mesh with four blocks around the blade is used to enable a high-quality grid distribution around the profile leading and trailing edges, while an H-type mesh with five additional blocks is used for the inflow, pas- 
sage and outflow sections to ensure good resolution of the incoming flow, the wake, and to enable the use of pitchwise periodic boundary conditions.

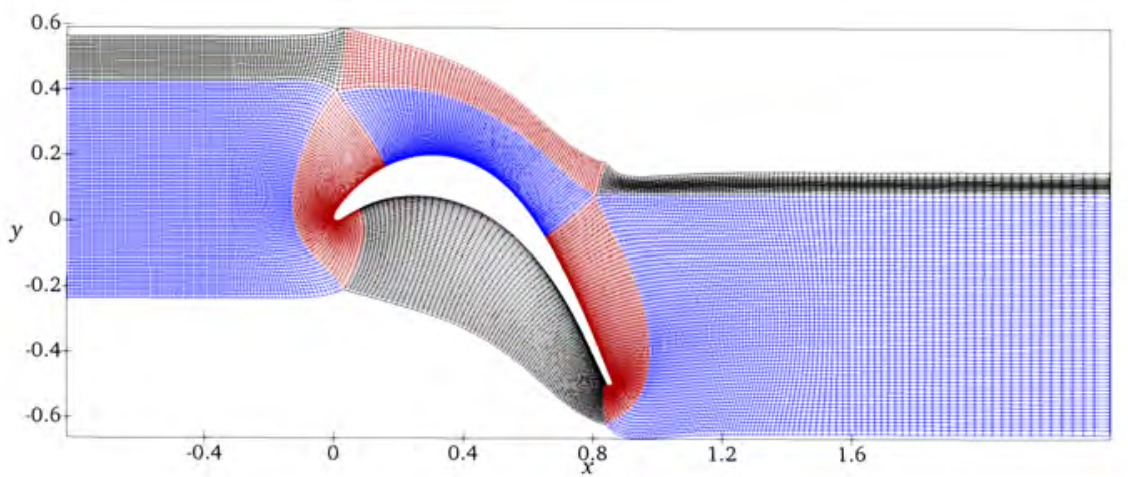

Fig. 7 Block topology and grid distribution for LPT case study. Case shown is grid G1 from table 2, with only every third grid point shown in each direction for clarity.

\subsection{T106A case study}

The tests are performed for a linear T106A cascade operating at an isentropic exit Reynolds number $\mathrm{Re}_{i s}=59,815$ and an isentropic exit Mach number of $\mathrm{Ma}_{i s}=$ 0.405 (see Sandberg et al. [117] for more details). The turbulence length scale is about $5 \%$ chord length and the turbulent intensity is $3.8 \%$. The operating conditions used are similar to an experiment conducted by Stadtmüller [134]. While the experimental case available at this low Reynolds number does not have a significant level of inlet turbulence the turbulence intensity for the case considered has been raised to $3.8 \%$ which is in the range of typical engines $(3-8 \%$ [2]). The spanwise extent of the domain is $20 \%$ true chord. Additional simulations with up to $60 \%$ chord have been conducted showing virtually identical results and thus implying that the spanwise domain size originally chosen was sufficient. If not otherwise stated all lengths, velocities and densities have been nondimensionalized with true chord length, inlet velocity magnitude and inlet density, respectively.

A total of eight grids were used to perform a grid sensitivity study, with the main characteristics summarized in Table 2. Grids G1 to G3 were DNS grids used to investigate the influence of the mesh size close to the blade on the loading and boundary layer properties. The spacing in the wall normal direction was roughly halved for each refinement level and the number of points in the spanwise direction was doubled from G1 to G2 and increased by another 50\% from G2 to G3. The number of 


\begin{tabular}{|c||c|c|c|c|}
\hline Grid & $n_{p}$ & $n_{z}$ & $n_{\text {tot }} \times 10^{6}$ & $y^{+}$ \\
\hline L1 & 85,248 & 34 & 2.7 & 2.4 \\
L2 & 85,248 & 34 & 2.7 & 1.0 \\
\hline G1 & 274,176 & 66 & 18.1 & 1.5 \\
G2 & 581,760 & 130 & 75.6 & 0.6 \\
G3 & $1,179,648$ & 194 & 200 & 1.0 \\
\hline D1 & 248,400 & 98 & 24 & 0.9 \\
D2 & 993,600 & 130 & 130 & 0.9 \\
D3 & $3,463,168$ & 194 & 672 & 0.9 \\
\hline
\end{tabular}

Table 2 Grids used for the grid-resolution study of linear T106A LPT cascade at $R e_{i s}=60,000$ and $M_{i s}=0.4$. Grids L1 and L2 denote LES grids, G1, G2 and G3 denote DNS grids varying wall resolution and grid D1, D2, D3 are DNS grid varying wake resolution. $n_{p}$ is the number of grid points for the axialpitchwise plane, $n_{z}$ denoted number of spanwise collocation points (over $20 \%$ axial chord span), $n_{t o t}$ is total number of points and $y^{+}$is maximum wall normal spacing on suction side.

points around the blade was increased from 864 to 1,296 and 1,824 for grids G1 to G2 and G3, respectively. This resulted in an increase of points around the blade of about $50 \%$ for each refinement level with most of the refinement clustered in the adverse pressure gradient region on the suction side where the majority of profile losses are expected to grow, and close to the trailing edge on the pressure side where transition might occur.

Grids L1 and L2 were based on grids G1 and G2, but with roughly double the grid spacing in each direction of the axial-pitchwise plane and with half the number of collocation points in the spanwise direction to be used for large eddy simulations with the same high-fidelity code. The wall adapting local eddy viscosity (WALE) model [21] was used for modeling of the sub-grid scale stresses with the standard coefficient of 0.325 .

Finally, another set of grids with increased resolution in the wake region was generated. The cell count in the downstream wake region was substantially and progressively increased while attempting to not further reduce the size of the smallest cells at the blade surface, which due to the use of an explicit time integration scheme dictates the time step of the simulation. With the grid counts shown in table 2 the non-dimensional grid spacings, normalized with blade chord, $\Delta=\sqrt{\delta \xi^{2}+\delta \eta^{2}}$ at the measurement location in the wake region, 0.24 axial chords downstream of the blade trailing edge, are approximately $3.3 \times 10^{-3}, 1.6 \times 10^{-3}$ and $0.7 \times 10^{-3}$ for grids D1, D2 and D3, respectively. Here, $\delta \xi$ and $\delta \eta$ are the grid spacings in the mapped computational space for the axial and pitchwise directions, respectively.

Figure 8 gives a visual impression on the capabilities of the coarsest and finest grids tested, i.e. L1 and D3, respectively, to resolve turbulent flow structures. The same iso-surface levels of the second invariant of the velocity gradient tensor, $Q=$ 100 , are chosen for both cases to allow for direct comparison. Overall, grid D3 appears to support a much greater amount of turbulent structures than grid L1. This is in particular the case for the inflow region where the nearly isotropic turbulence injected into the domain can be observed, which then undergoes strong acceleration and stretching resulting in lengthy streamwise orientated structures in the passage. For grid L1, the upstream turbulence is hardly visible and there are only few structures in the favorable pressure gradient part of the passage while the same type of 


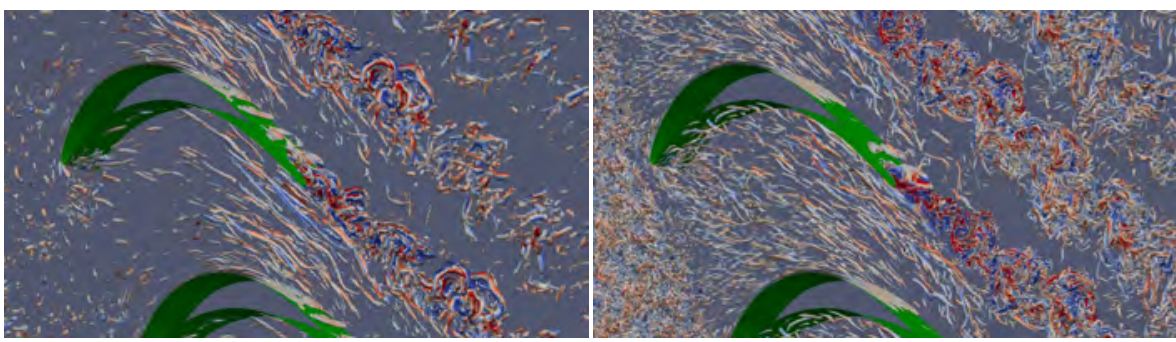

Fig. 8 Instantaneous isosurfaces of the second invariant of the velocity gradient tensor $Q=100$, colored by the spanwise velocity component (range -0.1 to 0.1 ) on grids L1 (left) and D3 (right)

long structures appear in the passage downstream of the suction peak as for grid D3. This indicates that although the grid is too coarse to resolve the small structures close to the leading edge region, it is sufficient for the resolution of the larger structures that result from the strong acceleration of the inflow turbulence. These structures strongly interact with the suction side boundary layers and as the next figures will show, being able to capture them allows the simulations to produce the correct loading and boundary layer properties. The much higher resolution of grid D3 also supports a much wider range of turbulence scales in the wake region. Whether this has an impact on the wake loss and turbulence quantities in that region will be discussed in the following.

In order to assess if grid convergence is achieved results for $\mathrm{L}$ and $\mathrm{G}$ grids are compared in figure 9 for several properties commonly used to assess the accuracy of numerical simulations. The pressure distributions are virtually identical for all grids, especially in the key peak suction and trailing edge regions, and the wall shear stress on the suction side, with only the last $20 \%$ chord shown to highlight the separation bubble, only shows negligible differences between the different grids. The kinetic wake loss is also compared between cases L1, L2 and G3 in figure 9, showing only very subtle differences. This indicates that for blade quantities and the wake loss, likely to be of most interest to designers, even the coarsest grids used for LES provide good accuracy.

In figure 10 several Reynolds stress components are compared in the wake region, for a measurement plane of 1.1 axial chords, i.e. 0.24 axial chords downstream of the blade trailing edge. This distance was chosen for the wake to fully develop and thus rigorously test whether the different grids allow for correct wake mixing. All normal Reynolds stress components and the shear stress component show only insignificant differences between the different DNS grids. Note that the LES grids L1 and L2 were by design significantly coarser in the wake region and thus the resolved Reynolds stress profiles, not shown here for clarity, exhibit some deviation from the DNS data. Thus it can be concluded that if second-order moments such as Reynolds stress profiles are of interest, a grid like G1 will produce grid-converged solutions.

Finally, the grid convergence is assessed using turbulence kinetic energy (TKE), the production of TKE and the dissipation of TKE as metrics. These are quantities of interest, for example, for turbulence model assessment and modelling, as well as for aerodynamic performance as the production of TKE is proportional to the entropy 

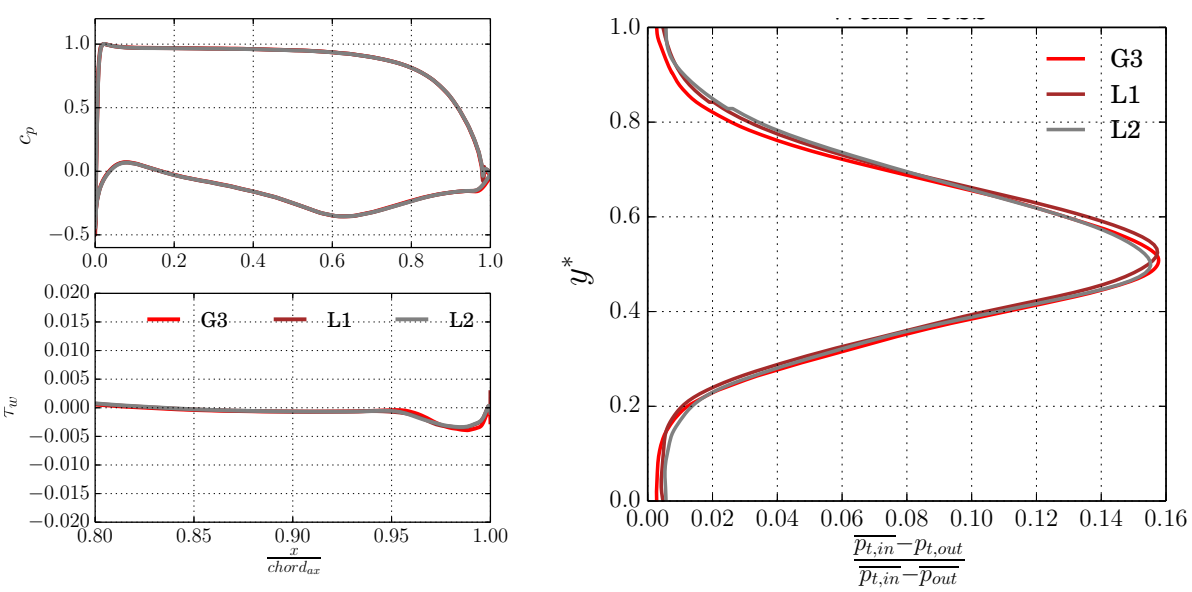

Fig. 9 Pressure distribution around the blade and wall shear stress on aft suction side (left) and wake loss profile (right) for grids L1, L2 and G3
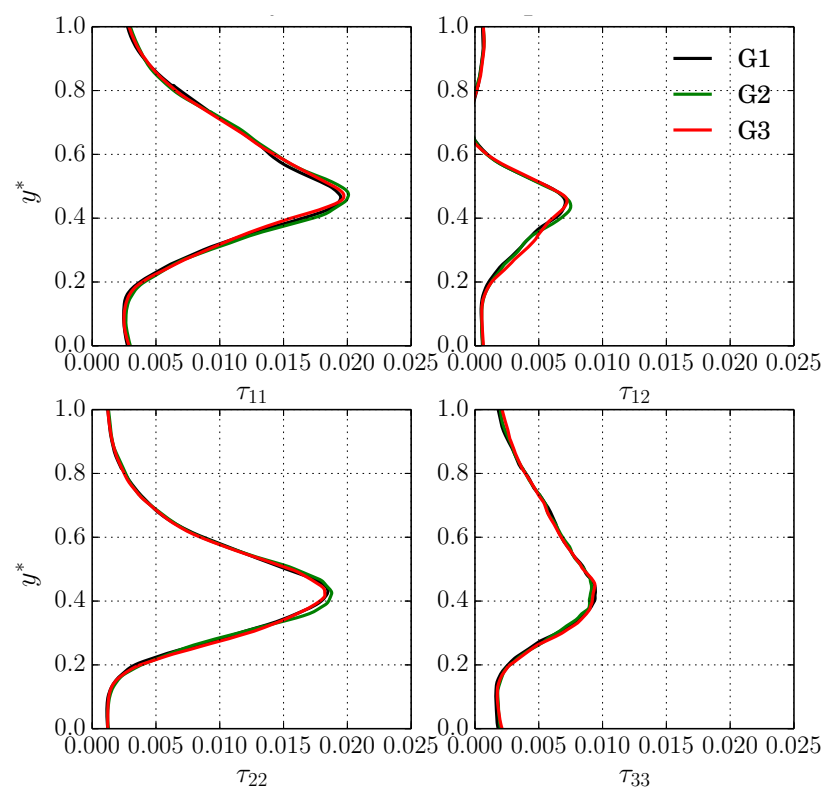

Fig. 10 Reynolds stress components for grids G1, G2 and G3 at 0.24 axial chords downstream of trailing edge

rate of change. In the wake the level of TKE and the production of TKE are dominated by large flow scales, thus these two quantities are unlikely to be much affected by the increasing resolution in grids D1 to D3, given that D1 already is sufficiently fine to produce grid converged Reynolds stress profiles. However, the dissipation of TKE is a viscous process and thus highly dependent on the accurate representation 

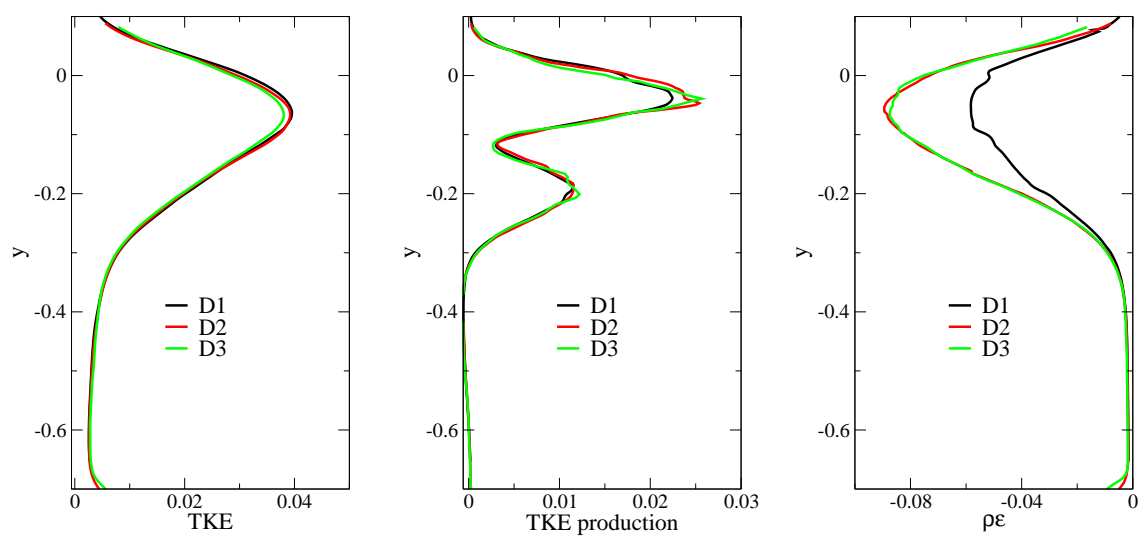

Fig. 11 TKE, TKE production and $\varepsilon$ in wake for grids D1, D2 and D3 at 0.24 axial chords downstream of trailing edge

of the smallest turbulence scales, or the Kolmogorov scales discussed in section 2 . Therefore, the dissipation of TKE serves as a much more stringent measure of grid convergence. The results shown in figure 11 are again presented for an axial position of 0.24 axial chords downstream of the trailing edge.

The three grids show reasonable agreement between each other in terms of TKE $(k)$ and its production, with the solutions on D1 exhibiting only slight differences to the other two grids. In contrast, the dissipation of TKE shows a different behaviour where grids D2 and D3 show reasonable agreement between each other while the magnitude of D1 is significantly smaller. The peak in dissipation of TKE is only about $60 \%$ of the corresponding value on the other grids.

In summary, the results presented in this section demonstrate that different resolution requirements are needed to achieve grid independence of different quantities. Ultimately, the required numerical discretization quality thus depends on the purpose of the simulations, i.e. what quantities are of interest. If mean flow properties, such as loading, skin friction, or even kinetic wake loss are of interest, grids with the quality of L1 are sufficient to produce grid independent results. If second-order statistics are of interest, grids like G1 are recommended. To obtain grid-independent results of dissipation of turbulence kinetic energy, as would be the case if the data were to be used to enhance turbulence models (see section 7), a grid like D2 is required. The latter case is what, strictly speaking, deserves to wear the label 'DNS'.

\subsection{Entropy based simulation quality assessment}

To establish guidelines for what simulation fidelity, in terms of grid resolution and order-of-accuracy, is required to correctly capture entropy losses, Przytarski \& Wheeler [109] established that the entropy generation rate based on the entropy flux could be used as a proxy for loss. Using a Taylor-Green vortex problem to represent fully turbulent flow, it was shown that a grid resolution with spacing of 4 and 2 times the 
Kolmogorov scale for 8th-order and 4th-order accurate schemes, respectively, was required for an accurate prediction of the entropy loss. Further, performing linear compressor cascade simulations with varying grid densities and freestream turbulence levels, they also demonstrated that losses can strongly depended on mesh quality through differences in transition location. Their study showed that an insufficient grid resolution in the laminar leading-edge region, where typically one would expect more benign resolution requirements, would suppress the boundary layer structures induced by the inflow turbulence and thus delay transition.

\section{Lower-order model maturation and improvement}

There are two avenues for high-fidelity simulations to have direct industrial impact. One is the use of the simulation data for physical insight, for example elucidation of detailed transition mechanisms or reliable calculation of entropy loss, that can be exploited by designers for improved aerothermal performance. However, there are not many examples of high-fidelity studies that have been conducted for a sufficiently wide parameter space to help guide designers, i.e. where design of experiments have been performed with high-fidelity approaches. The alternative is to use the highfidelity simulation results as gold- (or silver-) standard data bases that can be used for low-order model assessment and ultimately improvement.

Using high-fidelity data to test and develop turbulence models is not a novel concept. For example some of the early turbulent channel flow DNS data was also processed with the purpose of providing information for modelers, such as individual terms of the turbulence budget equations [72]. Rodi et al. [114] also used the same fully developed channel flow simulation data [55] to determine how turbulence is altered in terms of anisotropy, ratio of production rate versus dissipation, and length scale by the presence of a wall. This allowed the investigation of turbulence model assumptions and their accuracy in the proximity to a wall, an important effect in wall driven flows. The authors then 'manually' formulated a one-equation model capable of matching the wall normal velocity fluctuation and turbulent length scale from DNS at two Reynolds numbers. While the new model showed superior performance to others for channel flows, the authors also discovered that the improved accuracy in the wall proximity, i.e. for $y^{+}<30-40$, was unable to improve the overall prediction of the flow over a backward facing step.

Over the years, there have been many subsequent studies that have calibrated and modified turbulence models using high fidelity data, however, not always with the success desired. In the current contribution, we want to focus on the recent development of data-driven turbulence model assessment and development. This has come about as a result of the confluence of two key ingredients: (i) the availability of large amounts of data generated from high fidelity simulations, enabled by the dramatic growth of computing capabilities; and (ii) the proliferation of machine-learning and artificial intelligence approaches mainly driven by applications requiring image recognition and/or decision making, also enabled by the impressive developments in computing power, in particular accelerator-supported or based architectures. 
7.1 Review of data-driven model assessment and development

While machine learning algorithms, such as deep neural networks, have been used for a number of years in areas such as image or pattern recognition, the concept of using machine learning for the development of turbulence models is new. By trying to fit models to data, the machine learning techniques ultimately are not unlike classic curve-fitting methods that have been used to calibrate closures [17]. However, in machine learning jargon, the word 'train' is used instead of 'calibrate', as will also be done in the following. The crucial advantage of applying modern machine learning techniques to large data sets is that not only coefficients are trained, but also mathematical forms of closures can be inferred (trained). The aim is for the trained models to correctly represent the physics, albeit the closures might have no explicit connection to the exact turbulence equations. This modelling approach therefore can be classified as 'Openly Empirical' when following the taxonomy introduced by Spalart [131]. In fact, fully relying on a machine-learning algorithm to train a model from data could even be termed 'Maximally Empirical' [150].

The use of machine learning in a turbulence modelling context can be broadly divided into two areas: assessment and development. Uncertainty quantification, which aims to systematically determine why models fail and to provide confidence levels in the accuracy of the model predictions, falls into the first category. Several studies were conducted in the early 2000's that inspected high-fidelity databases to understand uncertainty and determine whether a given closure is applicable for a given flow topology $[47,120]$. Other methods of general uncertainty identification include passively solving the RANS equations using the mean velocity field extracted from DNS to assess the closures used [133]. In the context of machine-learning, the potential of three different algorithms (support vector machines, Adaboost decision trees, and random forests) to identify regions of high RANS uncertainty was investigated [69]. The uncertainty in the values of closure coefficients was determined with Bayesian methods [24] while a similar approach was chosen to quantify uncertainty in predicted flow quantities [160]. For a review of the uncertainties in RANS models the reader is referred to Duraisamy et al. [23]. However, to the authors' knowledge no machine-learning approaches have to date been applied to high-fidelity data of main gas path turbomachinery components for uncertainty quantification. In the following, we therefore want to focus on the use of machine learning for model development.

The use of machine learning for turbulence model development has been further categorized into studies that can demonstrate success in an a-priori or a-posteriori sense. In the prior case, the resulting models can be shown to approximate the training or other test data well. An example would be showing that a stress-strain closure model fits the turbulence stress from a high-fidelity data set well when applied to the strain field extracted from the high-fidelity data. It is assumed that having minimized a particular cost function will produce improved CFD predictions, although as will be shown later, this is not necessarily true. For models to be developed and tested in an $a$ posteriori sense means conducting full CFD analysis with the new model producing improved results on previously (to the model) unseen cases. Although this is much harder to achieve, it is the authors' opinion that it is the only route for machine-learnt models to have industrial impact. 
In the last few years, several different machine-learning techniques have been used. These can be approximately categorized into two groups. One group contains methods such as neural networks that have been applied to secondary flow prediction [68] and random forests [91], used for jet-in-crossflow studies. Gaussian processes have modeled corrective terms for channel flow [98]. Here correction parameters were learnt and added to the model transport equations. However, these were functions of space and thus any a-posteriori demonstration was limited to an identical geometry. More complex regression methods that alter more than the coefficients of a particular model have also been demonstrated. For example, deep neural networks have been used to model the Reynolds stress [67]. Although good a-priori agreement with reference data was found, a-posteriori demonstration required the matrix of Reynolds stresses at each grid point to be inserted into the solver, constraining the success of the model to the same geometrical configuration. One study also successfully extracted functional forms for intermittency transport to model bypass transition $[22,166]$. For heat-transfer problems random forest regression has been applied to model the turbulent thermal diffusivity, for a gradient-diffusion model in the scalar transport of temperature [91]. These approaches provide the user with a black box model and to the authors' mind, it is not entirely clear how portable the results obtained from those approaches are and whether they can easily be inserted into CFD codes. However, recent work has partly addressed this by also attempting to interpret a machine learning decision process by analyzing the model features and determining their importance [90]. Edeling et al. [25] have used Bayesian methods to modify model coefficients for a full a-posteriori demonstration and showed encouraging results. However, this approach proved to be computationally very intensive.

The other group is based on gene expression programming [28] (GEP), a form of evolutionary algorithm. The application of GEP to turbulence modeling was first reported in Weatheritt \& Sandberg [149], where a new stress-strain relationships for separated flow was presented. The GEP algorithm is a form of symbolic regression, such that the result of the high-fidelity data-fitting are tangible mathematical equations that are easy to manipulate, are transparent to the human user and, crucially, can be inserted into CFD solvers for no extra algorithm cost [150]. GEP has recently been applied to high-pressure turbines, where a-priori model training showed improvement over the linear model [148]. Basing their study on high-fidelity data of a jet-in-crossflow, Weatheritt et al. [151] compared neural networks and GEP approaches to regress non-linear stress-strain relationships and showed very similar predictive performance, yet the GEP optimization was at a fraction of the cost.

To close this short, non-exhaustive review, several different machine-learning strategies have recently been presented for turbulence model assessment, or uncertainty quantification, and development, all with different strengths and weaknesses. The review paper by Duraisamy et al. [23] provides additional information for the interested reader. In the next section, we present the application of one of those approaches for the development of models with improved predictive accuracy for lowand high-pressure turbine wake flows. 


\subsection{Data-driven model development for LPT and HPT}

The development of novel turbulence models with better predictive accuracy, ideally across the operating range of interest to designers, requires two ingredients. Reliable data for a sufficiently large sample of the design space, and machine learning algorithms that are capable of building the desired models. In this section we summarize recent efforts on applying the GEP approach [149] to the data that has been obtained from recent high-fidelity simulations [86, 103, 117, 152] of low- and high-pressure turbine cascades.

The modelling assumption in RANS that has been the initial focus on the closure training is the commonly used Boussinesq approximation

$$
\tau_{i j}=\frac{2}{3} k \delta_{i j}-2 \nu_{t} S_{i j}
$$

This approximation dictates that deviations from the isotropic part $\frac{2}{3} k \delta_{i j}$, where $k$ is the turbulent kinetic energy, are linear in the strain rate tensor $S_{i j}$. The constant of proportionality, the eddy viscosity $\nu_{t}$, in most cases will be obtained from one or two transport equations [66]. Addressing this closure approximation first was motivated by the fact that the Reynolds stress term appears both in the RANS momentum equations, more precisely its divergence, and in the production terms of the turbulence transport equations. Furthermore, assessment of the Boussinesq assumption against high fidelity data has shown it to be a significant source of error [105].

Using high fidelity data, one of the error sources can be quantified by considering the alignment of the strain rate tensor and the anisotropy tensor, computed as follows [120]

$$
\gamma=-\frac{a_{i j}^{D N S} S_{i j}}{\sqrt{a_{m n}^{D N} a_{n m}^{D N S} S_{p q} S_{q p}}}, \quad-1<\gamma<1,
$$

with

$$
a_{i j}^{D N S}=\tau_{i j}^{D N S}-\frac{2}{3} k^{D N S} \delta_{i j}=-2 \nu_{t}^{D N S} S_{i j}^{D N S},
$$

where $\tau_{i j}$ is the Reynolds stress tensor, $k$ the turbulence kinetic energy, $\nu_{t}$ the turbulence viscosity and $S_{i j}$ the strain rate tensor. A value of -1 implies that the strain rate and anisotropy tensor are aligned in the opposite direction. A value of 0 implies no alignment between the stress and strain rate tensor. Finally, a value of +1 indicates strong alignment between the anisotropy and strain rate tensor.

In Fig. 12, it can be seen that there exists a regions of poor alignment close to the trailing edge and along the red dashed lines. Thus, the objective of the GEP framework is to add terms to the linear Boussinesq approximation, to form a non-linear constitutive stress-strain relationship that improves the alignment $\gamma$. Such turbulence closures, also known as explicit algebraic stress models, have been developed in the past (e.g. [32, 144]) invoking a weak equilibrium hypothesis [113] to express a tensor basis $V_{i j}^{(k)}$ and scalar invariants $I_{k}$ for the anisotropy tensor $a_{i j}$ [107]. 


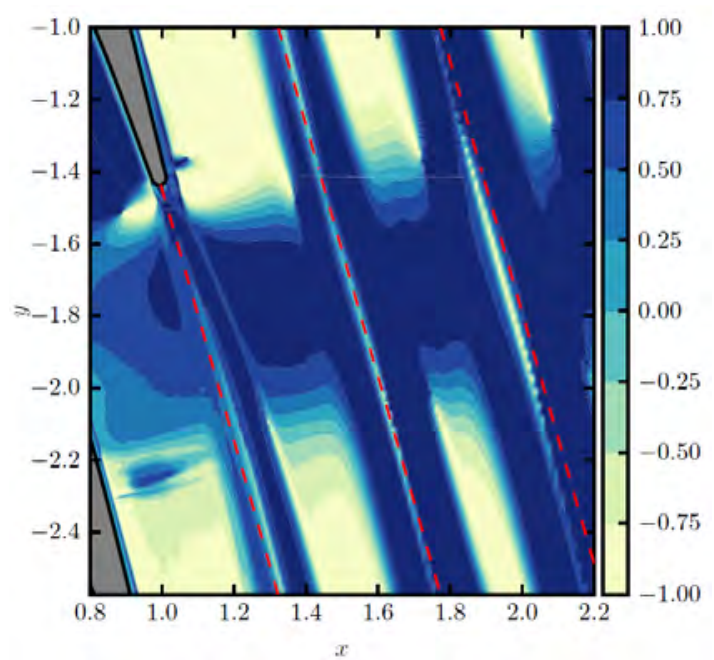

Fig. 12 Alignment of turbulent stress anisotropy with negative strain, $\gamma$, using DNS data of a linear LS89 HPT cascade at $R e=570,000$ [152] in the wake region of the blade.

$$
\tau_{i j}=\frac{2}{3} k \delta_{i j} \underbrace{-2 \nu_{t} S_{i j}}_{\text {linear Bouss. }}+\underbrace{2 k \sum_{k=1}^{4} \zeta_{k} V_{i j}^{k}}_{\text {additional non-linear terms }} .
$$

This relates the Reynolds stress tensor $\tau_{i j}$ to its trace $2 k$, strain rate tensor $S_{i j}$ and additional non-dimensional terms. $V_{i j}^{k}$ are basis functions formed from polynomials of non-dimensional strain $s_{i j}=\tau S_{i j}$ and rotation rate $w_{i j}=\tau \Omega_{i j}$ tensors

$$
\begin{aligned}
& V_{i j}^{(1)}=s_{i j}, \quad V_{i j}^{(2)}=s_{i k} w_{k j}-w_{i k} s_{k j}, \\
& V_{i j}^{(3)}=s_{i k} s_{k j}-\frac{1}{3} \delta_{i j} s_{m n} s_{n m}, \\
& V_{i j}^{(4)}=w_{i k} w_{k j}-\frac{1}{3} \delta_{i j} w_{m n} w_{n m},
\end{aligned}
$$

The coefficients $\zeta_{k}$ are functions of the non-dimensional invariants $I_{k}$ produced from $s_{i j}$ and $w_{i j}$, of which there are only two for statistically $2 \mathrm{D}$ flows,

$$
I_{1}=s_{m n} s_{n m}, \quad I_{2}=w_{m n} w_{n m} .
$$

$\tau$ is the turbulence model timescale that is obtained by solving the turbulence transport equations for $\omega$ or $\epsilon$, depending on which underlying baseline model will be used for the non linear EARSM models. Thus the turbulence time scale can either be computed from the specific dissipation rate $\tau=1 / \omega$, or as $\tau=k / \epsilon$.

The baseline RANS solution thus is obtained from setting $\zeta_{k}=0$ and the task of the machine learning algorithm is to use the high-fidelity data to find the coefficients

$$
\zeta_{k}=\zeta_{k}\left(I_{1}, I_{2}\right), \quad k=1, \ldots 4,
$$


that are suitable for the flow under consideration. It is important to note that because the new models are a linear combination of basis functions derived from the velocity gradient tensor, Galilean invariance is inherently enforced and does not need to be imposed as an additional constraint, as is the case in other data-driven model development approaches[68].

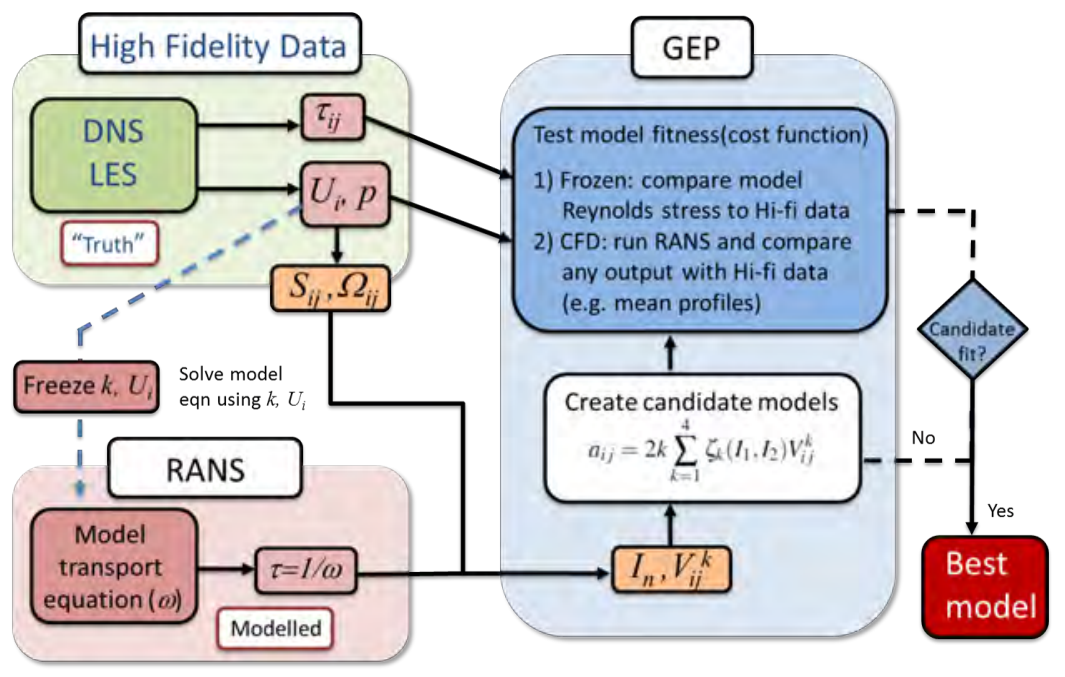

Fig. 13 Schematic of GEP model development framework.

Figure 13 summarizes the model development framework. From the high-fidelity data the mean flow field, the turbulence kinetic energy, and the Reynolds stress, strain rate and vorticity tensors are extracted. In order to non-dimensionalize the basis functions and scalar invariants for the model training, a turbulent timescale, $\tau$ is required. Extracting this timescale directly from the high-fidelity data has proven difficult and resulted in very noisy fields. Therefore, the turbulent time scale is obtained by solving a transport equation for $\varepsilon$ or $\omega$, 'freezing' the high-fidelity velocity and turbulence kinetic energy field. This also has the advantage that the model to be developed will be based on the high-fidelity data while being consistent with the transport equation used to determine $\tau$, provided the same transport equation is then used for subsequent CFD calculations with the trained model. Note that this implies that a different Reynolds stress closure will need to be trained for each set of underlying turbulence transport equations to be used. For more information on this 'frozen' technique, see previous works [99, 133, 150].

Once the turbulence time scale is known the scalar invariants and basis functions $I_{n}$ and $V_{i j}^{(k)}$ can be calculated from the high-fidelity data and the GEP algorithm is tasked to create a number of candidate models (how many is determined by the user). The so-called fitness is then evaluated for each of these candidate models. This can be done in two was. Firstly, a cost function is evaluated, which in the cases presented below is the squared error between the anisotropy tensors of the model 
and the high-fidelity data (first option in Figure 13). We term this 'frozen training' as the model fitness is evaluated using the frozen velocity field from the high-fidelity data set. This method is unable to account for any additional errors introduced by the turbulence transport equations of the RANS method used. In the second approach, the candidate models are implemented into a CFD solver and a RANS calculation is performed for each of the models [167]. This approach has multiple advantages: the cost function used to evaluate whether the new model is successful is not limited to turbulence quantities or terms that appear in the RANS equations but can be mean variables of interest, e.g. kinetic wake loss or shear stress on the blade, etc. Also, any additional errors introduced by other modelling assumptions of the RANS equations are accounted for. However, there are two downsides to this approach. Firstly, the resulting models are more difficult to interpret as they might try to compensate for multiple form errors of the turbulence model. Secondly, and much more restricting, this method is computationally much more expensive as CFD solutions are required for the candidate models. This might even be prohibitive if models are to be developed for complex three-dimensional geometries or unsteady RANS where each CFD run requires substantial time.

With 'frozen' approach the cost function does not entirely capture transport effects and the training can be called 'local'. If the quality of the model is determined by a RANS calculation the training process is 'non-local' as the cost function will explicitly incorporate transport effects.

Once the model fitness has been evaluated, it is checked whether the resulting error is less than a prescribed threshold. Should this be the case, the model is output and can then be used for future CFD runs. If the fitness is less than desired, the GEP algorithm creates new candidate models using the previous generation of models, disregarding the least fit models, mating the most fit ones and introducing mutations [149]. The fitness of these new models is then evaluated and the process continues until suitable models are found or a prescribed number of model generations has been reached.

Using the 'frozen' approach just outlined, for the T106A LPT cascade described in Sandberg et al. [117] the GEP approach applied to the DNS data produced the following model

$$
\begin{aligned}
\tau_{i j}=\frac{2}{3} k \delta_{i j} & -2 \nu_{t} S_{i j}+2 k[ \\
& +\left(3 I_{1}+3.205 I_{2}-1.2665\right) V_{i j}^{1} \\
& +\left(-4.0 I_{1} I_{2}^{2}-12 I_{1} I_{2}-8 I_{1}+4 I_{2}^{3}+20 I_{2}^{2}+32 I_{2}+16\right) V_{i j}^{2} \\
& \left.+\left(-8 I_{1}+48\right) V_{i j}^{3}\right],
\end{aligned}
$$

when using the 'frozen training' approach. Using only the leading order term of the trained model, the linear part of the Reynolds stress tensor can be written as

$$
\begin{aligned}
\tau_{i j} & =\frac{2}{3} k \delta_{i j}-2 \nu_{t} S_{i j}-2 k\left[1.2665 \tau S_{i j}\right] \\
& =\frac{2}{3} k \delta_{i j}-2(1+1.2566) \nu_{t} S_{i j} .
\end{aligned}
$$


Therefore the trained model increases the turbulence diffusion by roughly $125 \%$. Additionally, nonlinear terms based on $V_{i j}^{2}$ and $V_{i j}^{3}$ are added. In contrast, when choosing the CFD-supported training approach, the new model takes the form

$$
\begin{aligned}
\tau_{i j}=\frac{2}{3} k \delta_{i j} & -2 \nu_{t} S_{i j}+2 k[ \\
& -\left(2.3-0.089 I_{2}+I_{2}^{2}-0.15 I_{2}^{3}\right) V_{i j}^{1} \\
& +\left(-1+I_{1} I_{2}^{2}-I_{1}^{2} I_{2}\right) V_{i j}^{2} \\
& \left.+\left(1.903 I_{2}-I_{1} I_{2}+I_{1}^{2} I_{2}+I_{2}^{3}\right) V_{i j}^{3}\right] .
\end{aligned}
$$

In this case, the model diffusion is increased by $230 \%$, thus significantly more than in the 'frozen training' approach. At the same time, the leading order coefficients of the nonlinear basis functions have considerably reduced. The resulting CFD predictions using these models are shown in Fig. 14. The left figure shows the kinetic wake loss at $25 \%$ chord downstream of the trailing edge obtained from RANS conducted of the linear T106A LPT cascade configuration at $R e=60,000$, i.e. the case the new models were trained on. The baseline model using the linear stress-strain relationship overpredicts the kinetic wake loss significantly and underpredicts the width of the wake. The model trained using the frozen approach (Eg. 9) reduces the peak amplitude and shows a better spread of the profile. Thus, the additional diffusion introduced by the new model improves the mean flow solution. The CFD-supported training shows an even better agreement with the reference data, particularly in terms of the peak amplitude of the kinetic wake loss. In order to test whether this new model
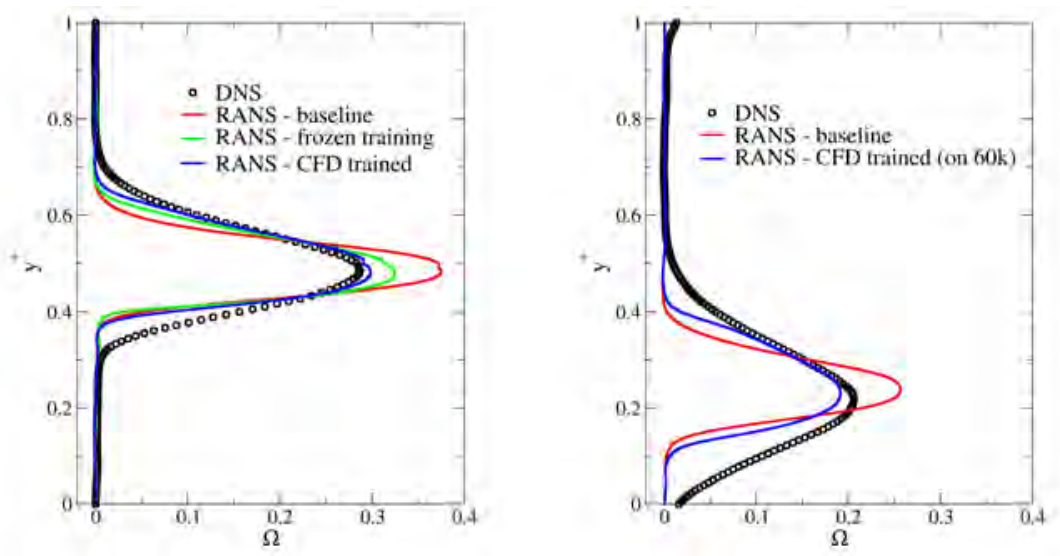

Fig. 14 Kinetic wake loss for LPT cascade with T106A profile at $R e=60,000$ (left) and $R e=100,000$ (right), obtained from DNS [86], and $k-\omega$-SST model with linear (baseline) and GEP trained non-linear stress-strain relationships.

(Eqn. 11) also does well on cases it was not trained for, it is also applied to a higher Reynolds number case $(R e=100,000)$ that features a qualitatively different flow field in that the separation on the suction side of the LTP blade is closed rather than 
open as in the $R e=60,000$ case [1]. The model trained on the $R e=60,000$ case also shows significant improvement of the kinetic wake loss for the $R e=100,000$ case.

In the case of the linear LS89 HPT cascade, using the setup and data described in Pichler et al. [103], the frozen training results in the model [148]

$$
\begin{aligned}
\tau_{i j} & =\frac{2}{3} k \delta_{i j}-2 \nu_{t} S_{i j}+2 k[ \\
& -\left(1.334-0.438 I_{1}-2.653 I_{2}-0.0102 I_{1}^{2}+1.021 I_{2}^{2}-12.280 I_{1} I_{2}\right) V_{i j}^{1} \\
& +\left(0.573-1.096 I_{1}+8.985 I_{2}-0.1102 I_{1}^{2}+2.876 I_{2}^{2}+90.633 I_{1} I_{2}\right) V_{i j}^{2} \\
& \left.+\left(12.861-25.094 I_{1}+6.449 I_{2}+1.020 I_{1}^{2}-304.979 I_{1} I_{2}-184.519 I_{2}^{2}\right) V_{i j}^{3}\right] .
\end{aligned}
$$

As in the LPT case, the GEP-trained model diffusion is increased over the linear baseline model by more than $100 \%$. However, when choosing the CFD-supported approach, a further dramatic increase, $700 \%$, in turbulence model diffusion is obtained.

$$
\begin{aligned}
\tau_{i j}=\frac{2}{3} k \delta_{i j} & -2 \nu_{t} S_{i j}+2 k[ \\
& -\left(I_{1} I_{2}+7\right) V_{i j}^{1} \\
& +\left(I_{1}^{3} I_{2}+2 I_{1}^{2} I_{2}-I_{1}^{2} I_{2}^{2}+2 I_{1} I_{2}-2 I_{2}+I_{1}-2\right) V_{i j}^{2} \\
& \left.+\left(I_{1}+3 I_{2}-1.62\right) V_{i j}^{3}\right] .
\end{aligned}
$$

As can be seen in Fig. 15, this large additional diffusion factor is indeed needed in order to predict the (nearly) correct amplitude of the kinetic wake loss for these high Reynolds number cases. Relying only on the frozen training in this case does not significantly improve results, presumably because driving the anisotropy tensor to match the training data does not guarantee better prediction of mean flow quantities once full CFD calculations are performed. The new model (Eq. 13) was also used to run RANS of the higher Reynolds number case, $R e=1,100,000$, and a significant improvement of the kinetic wake loss prediction was again observed.

Thus, the GEP approach presents itself as a promising avenue for the development of turbulence models with improved accuracy that can be easily implemented into existing CFD solvers. Such a method that demonstrates robust accuracy improvements over a broader parameter space can help with generating more industrial impact from high-fidelity data.

\section{Summary and Outlook}

The exponential growth of papers on DNS and LES of turbomachinery relevant flows is testament to the growing interest of both academia and industry on this subject. The so-called high-fidelity simulations are indeed shedding light on fundamental aero-thermal phenomena happening in turbines and compressors, and they have also proved capable of helping the development of lower-order models, the use of which 

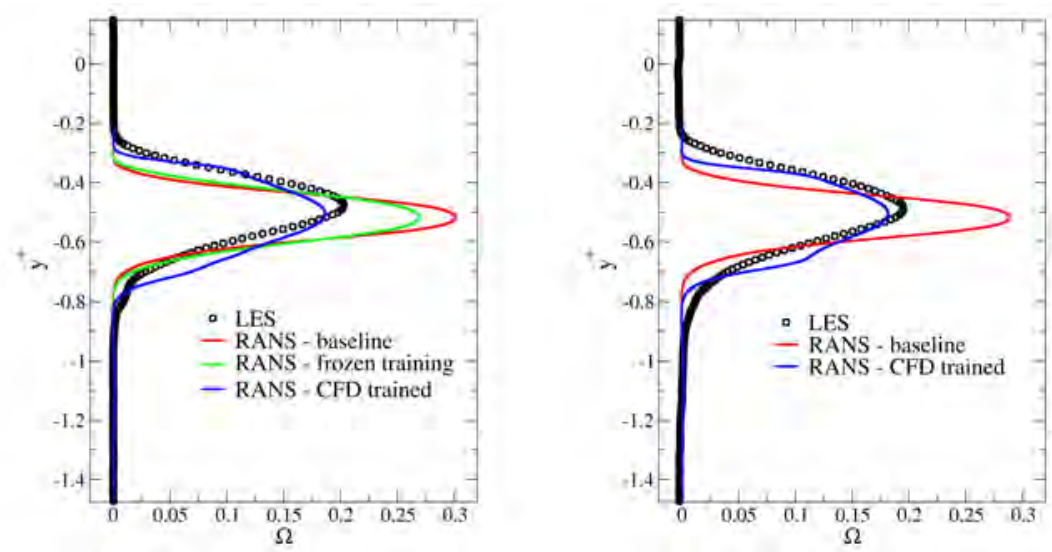

Fig. 15 Kinetic wake loss for HPT cascade with LS89 profile at $R e=570,000$ (left) and $R e=$ $1,000,000$ (right), obtained from LES [103], and $k-\omega$-SST model with linear (baseline) and GEP trained non-linear stress-strain relationships.

is dictated by the still excessive computational effort of LES if used for design iterations.

The complexity of realistic flow configurations, that should include clearance, cavities, extractions, high Reynolds and the combination of low and high Mach numbers, together with the need to consider variable operating conditions mean that the practical and routine use of $L E S$ to complete design iterations is currently not practical in industry. However, this must not be perceived as an unsurmountable obstacle as further advances in both computational power and post-processing techniques will lead to increased adoption of the practical application of LES in industry. For example, high-fidelity CFD is already used to run DoEs to investigate fundamental design parameters, like flow coefficient, reduced frequency, axial gaps, profile loads, or to determine the details of axial compressor stall mechanism, to name a few. In this case the computational domain focuses exclusively on the physics and the regions of interest in a sort of scaled-down investigation.

Therefore, DNS and LES are becoming a very accurate virtual test-rig for a growing range of design related problems that had been so far investigated mostly by using scaled down experimental rigs in simplified operating conditions, a typical example of which are linear compressor and turbine linear cascades. Despite the formidable improvements in measurement techniques, often experimental tests can identify the "what", while high-fidelity CFD is capable of explaining the "why" thanks to the higher degree of resolution and depth. The consequent findings can be used to improve existing design correlations with a physical foundation and accuracy superior to correlations based solely on experiments. In summary, the numerically produced data allows to discern the different physical processes that impact aero and thermal performance, forced response, flutter, and life.

Another promising short term application of both DNS and LES is to provide reference data sets to improve lower order models with the aid of Artificial Intelligence (AI). As pointed out by Lumley [71], a general constitutive law that will hold valid in 
the entire flow field of a turbomachine is impossible to achieve due to the very wide range of time and length scales. Nevertheless, machine-learning techniques are capable of determining the portions of the computational domain where different closure amendments should be applied. In other words, it appears that high-fidelity simulations with the aid of AI can indicate model improvements to cure known deficiencies of RANS and revive turbulence model development, that has been stagnating over the last decades. In particular, the development and calibration of non-linear constitutive laws of the type indicated by Gatski [32] can benefit from machine-learning, as witnessed by recent preliminary developments applied to wakes and effectiveness.

The very rich DNS and LES results cannot be postprocessed by using conventional techniques. Among the different methods used to extract the most out of highfidelity CFD datasets, POD deserves a special mention. Recent advances in this technique have shown how it is possible to associate each POD mode to a specific entropy content. Following this approach makes it possible to associate an entropy rate of change to each mode. For example, POD is capable to point at performance top offenders that a designer may concentrate on during design iterations.

The tremendous growth of computational power enables simulations that were unthinkable only few years ago. Paradoxically, the vast amount of data generated make it hard to scrutinize in detail the numerical data sets, that are literally gold mines in terms of physics content. Advances in improved and faster postprocessing methods, like the AI and POD approaches mentioned in this paper, are necessary to supplement phenomenological analysis with a more analytic approach. To fully exploit the valuable data sets, produced at significant cost in terms of both human and computing time, will also require a rethink of how to share such data. This will include agreed data standards and individual researchers dropping their idiosyncrasies, and other procedural and IP-related issues. Most likely, support from data science and informatics will be required to address this challenge through the development of protocols and platforms to share and mine new and old data.

Ensuring the quality of both DNS and LES simulations is also of paramount importance. High-fidelity simulations come with very stringent requirements in terms of resolution in both space and time, as well as boundary conditions. A proper DNS, with appropriate resolution of the boundary layers, critical in wall driven flows, as well as of the flow core, where a good portion of losses are generated due to bladerows interactions, is currently possible only for limited classes of flows. LES is indeed a valid alternative, but it is necessary to verify the impact of the sub-grid-scale model and errors induced by the numerical scheme or the grid resolution. Therefore, stringent quality checks are required to make sure the proper balance between modeling and resolving turbulence is achieved, and the results are not contaminated by spurious errors and thus reliable. Nevertheless, with continued increase in available computing power, high-fidelity simulations will unveil the detailed flow physics in increasingly complex and realistic main gas path turbomachinery component configurations, and thus their industrial impact will grow.

Acknowledgements The authors acknowledge funding for part of the work by General Electric and their permission to publish. RDS also acknowledges support from veski. Some of the presented data were obtained through INCITE and ALCC projects using resources of the Oak Ridge Leadership Computing 
Facility at the Oak Ridge National Laboratory, which is supported by the Office of Science of the U.S. Department of Energy under Contract No. DE-AC05-00OR22725. Additional computing time was provided through the Partnership for Advanced Computing in Europe (PRACE) and the UK Turbulence Consortium funded by the EPSRC under Grant No. EP/L000261/1, and a grant from the Swiss National Supercomputing Centre (CSCS) under project ID S622. Some work was also supported by resources provided by the Pawsey Supercomputing Centre with funding from the Australian Government and the Government of Western Australia.

\section{Compliance with Ethical Standards}

Conflict of Interests: The authors declare that they have no conflict of interest.

Funding: This work was partly funded by veski and by General Electric.

\section{References}

[1] Akolekar, H., Weatheritt, J., Hutchins, N., Sandberg, R., LASKOWSKI, G. \& MiCHELASSI, V. 2018 Development and Use of MachineLearnt Algebraic Reynolds Stress Models for Enhanced Prediction of Wake Mixing in LPTs. In ASME Turbo Expo 2018: Turbomachinery Technical Conference and Exposition, pp. GT2018-75447. American Society of Mechanical Engineers.

[2] ARndt, N. 1993 Blade Row Interaction in a Multistage Low-Pressure Turbine. ASME J. Turbomachinery 115 (1), 137.

[3] Arts, T and Lambert de Rouvroit, M And Rutherford, AW 1990 Aero-thermal investigation of a highly loaded transonic linear turbine guide vane cascade. A test case for inviscid and viscous flow computations. In Proc. of the ASME IGTI 90-GT-358.

[4] BAldwin, B. \& Lomax, H. 1978 Thin-layer approximation and algebraic model for separated turbulent flows. In 16th Aerospace Sciences meeting, p. 257.

[5] Bhaskaran, R., Jia, F., LASkOwski, G., WAng, Z. \& Paliath, U. 2017 Towards high-order large eddy simulation of aero-thermal flows for turbomachinery applications. In ASME Turbo Expo 2017: Turbomachinery Technical Conference and Exposition, pp. V02BT41A011-V02BT41A011. American Society of Mechanical Engineers.

[6] BhASKARAN, R. \& LELE, S. K. 2010 Large eddy simulation of free-stream turbulence effects on heat transfer to a high-pressure turbine cascade. Journal of Turbulence (11), N6.

[7] Bisset, D., AntoniA, R. \& BRitz, D. 1990 Structure of large-scale vorticity in a turbulent far wake. J. Fluid Mech. 218, 463-482.

[8] Braza, M., Perrin, R. \& Hoarau, Y. 2006 Turbulence properties in the cylinder wake at high Reynolds numbers. Journal of Fluids and Structures 22 (6-7), 757-771.

[9] Cassinelli, A., Adami, P., Montomoli, F. \& Sherwin, S. 2018 High fidelity spectral/HP element methods for turbomachinery. In ASME Turbo Expo 
2018: Turbomachinery Technical Conference and Exposition, pp. GT201875733. American Society of Mechanical Engineers.

[10] ChOI, H. \& MoIN, P. 2012 Grid-point requirements for large eddy simulation: Chapmans estimates revisited. Phys. Fluids 24 (1), 011702.

[11] Ciorciari, R., KiRIK, I. \& Niehuis, R. 2014 Effects of Unsteady Wakes on the Secondary Flows in the Linear T106 Turbine Cascade. ASME J. Turbomachinery 136 (9), 091010-091010-11.

[12] Coleman, G., GarbaruK, A. \& Spalart, P. 2014 Direct Numerical Simulation and Theories of Wall Turbulence With a Range of Pressure Gradients. 10th International ERCOFTAC Symposium on Engineering Turbulence Modelling and Measurements (ETMM10) pp. 261-276.

[13] Colonius, T. \& Lele, S. 2004 Computational aeroacoustics: progress on nonlinear problems of sound generation. Progress in Aerospace Sciences 40 (6), 345-416.

[14] Comte-Bellot, G. \& Corrsin, S. 1971 Simple Eulerian time correlation of full-and narrow-band velocity signals in grid-generated, isotropic turbulence. J. Fluid Mech. 48, 273-337.

[15] Cornelius, C., Biesinger, T., Galpin, P. \& Braune, A. 2013 Experimental and Computational Analysis of a Multistage Axial Compressor Including Stall Prediction by Steady and Transient CFD Methods. ASME J. Turbomachinery 136 (6), 061013-061013-12.

[16] CUI, J. \& TUCKER, P. 2016 Numerical study of purge and seconday flows in a low-pressure turbine. ASME J. Turbomachinery 139, 021007.

[17] DAlY, B. J. \& HaRlow, F. H. 1970 Transport equations in turbulence. Phys. Fluids 13 (11), 2634-2649.

[18] Denton, J. 1993 The 1993 IGTI Scholar Lecture: Loss Mechanics in Turbomachines. ASME J. Turbomachinery 115 (4), 621-656.

[19] Dickens, T. \& DAY, I. 2010 The Design of Highly Loaded Axial Compressors. ASME J. Turbomachinery 133 (3), 031007-031007-10.

[20] Duchaine, F., Dombard, J., Gicquel, L. \& Koupper, C. 2017 On the importance of inlet boundary conditions for aerothermal predictions of turbine stages with large eddy simulation. Computers \& Fluids 154, 60-73.

[21] Ducros, F., Nicoud, F. \& Poinsot, T. 1998 Wall-adapting local eddyviscosity models for simulations in complex geometries.

[22] Duraisamy, K. \& DURBin, P. 2014 Transition modeling using data driven approaches. Proceedings of the CTR Summer Program p. 427.

[23] Duraisamy, K., Iaccarino, G. \& XiaO, H. 2019 Turbulence modeling in the age of data. Ann. Rev. Fluid Mech. 51 (1).

[24] Edeling, W., Cinnella, P. \& Dwight, R. P. 2014 Predictive rans simulations via bayesian model-scenario averaging. J. Comp. Phys. 275, 65-91.

[25] Edeling, W., Cinnella, P., Dwight, R. P. \& Bijl, H. 2014 Bayesian estimates of parameter variability in the $\mathrm{k}-\varepsilon$ turbulence model. J. Comp. Phys. 258, 73-94.

[26] Engber, M. \& FotTner, L. 1996 The effect of incoming wakes on boundary layer transition of a highly loaded turbine cascade. In AGARD CONFERENCE PROCEEDINGS. AGARD. 
[27] Espinal, D., IM, H.-S. \& ZHA, G.-C. 2017 Full-Annulus Simulation of Nonsynchronous Blade Vibration Excitation of an Axial Compressor. ASME J. Turbomachinery 140 (3), 031008-031008-12.

[28] FERREIRA, C. 2001 Gene expression programming: A new adaptive algorithm for solving problems. Complex Systems 13 (2), 87-129.

[29] Fransen, R., Collado, E., Duchaine, F., Gourdain, N., Gicquel, L., Vial, L. \& BONNEAU, G. 2011 Comparison of RANS and LES in high pressure turbines. In 3me Colloque INCA, ONERA, Toulouse, France, Nov, pp. $17-18$.

[30] Garai, A., Diosady, L., Murman, S. \& Madavan, N. 2015 Dns of flow in a low-pressure turbine cascade using a discontinuous-galerkin spectralelement method. In ASME Turbo Expo 2015: Turbine Technical Conference and Exposition, pp. V02BT39A023-V02BT39A023. American Society of Mechanical Engineers.

[31] Garai, A., Diosady, L. T., Murman, S. M. \& Madavan, N. K. 2018 Scale-resolving simulations of bypass transition in a high-pressure turbine cascade using a spectral element discontinuous Galerkin method. ASME J. Turbomachinery 140 (3), 031004.

[32] Gatski, T. B. \& Speziale, C. G. 1993 On explicit algebraic stress models for complex turbulent flows. J. Fluid Mech. 254, 59-78.

[33] Germano, M. 1992 Turbulence: The filtering approach. J. Fluid Mech. 238, 325-336.

[34] Gourdain, N. 2013 Prediction of the unsteady turbulent flow in an axial compressor stage. Part 1: Comparison of unsteady RANS and LES with experiments. Computers \& Fluids 106 (5), 119-129.

[35] Gourdain, N., Gicquel, L. \& Collado, E. 2012 Comparison of rans and les for prediction of wall heat transfer in a highly loaded turbine guide vane. Journal of propulsion and power 28 (2), 423-433.

[36] Gourdain, N., Sicot, F., Duchaine, F. \& Gicquel, L. 2014 Large Eddy Simulation of Flows in Industrial Compressors: a Path from 2015 to 2035. Philos Trans A Math Phys Eng Sci. 372.

[37] Grimshaw, S., Pullan, G. \& Walker, T. 2015 Bleed-Induced Distortion in Axial Compressors. ASME J. Turbomachinery 137 (10), 101009-101009-9.

[38] Guleren, K., Turan, A. \& Pinabarsi, A. 2008 Large Eddy Simulation of the Flow in a Low-Speed Centrifugal Compressor. International Journal for Numerical Methods in Fluids 56 (8), 1271-1280.

[39] Halstead, D., Wisler, D., Okisshi, T., Walker, G., Hodson, H. \& SHIN, H.-W. 1997 Boundary layer development in axial compressors and turbines: Part 1 of 4 - Composite picture. ASME J. Turbomachinery 119 (1), 114 127.

[40] Hammer, F., SANDham, N. \& SANDBERG, R. 2018 The influence of different wake profiles on losses in a low pressure turbine cascade. International Journal of Turbomachinery, Propulsion and Power 3 (2), 10.

[41] HAMmer, F., SANDHAM, N. \& SANDBERG, R. 2018 Large eddy simulations of a low-pressure turbine - roughness modeling and the effects on boundary layer transition and losses. In ASME Turbo Expo 2018: Turbomachinery Tech- 
nical Conference and Exposition, pp. GT2018-75796. American Society of Mechanical Engineers.

[42] Harnieh, M., Gicquel, L. \& Duchaine, F. 2017 Sensitivity of large eddy simulations to inflow condition and modeling if applied to a transonic highpressure cascade vane. In ASME Turbo Expo 2017: Turbomachinery Technical Conference and Exposition, pp. V02BT41A043-V02BT41A043. American Society of Mechanical Engineers.

[43] Heinke, W., König, S., Matyschok, B., Stoffel, B., Fiala, A. \& HEINIG, K. 2004 Experimental investigations on steady wake effects in a high-lift turbine cascade. Experiments in Fluids 37 (4), 488-496.

[44] Hellstrom, F., Gutmark, E. \& Fuchs, L. 2012 Large Eddy Simulation of the Unsteady Flow in a Radial Compressor Operating Near Surge. ASME J. Turbomachinery 134 (5), 051006.

[45] Henderson, A., WAlKer, G. \& Hughes, J. 2005 The Influence of Turbulence on Wake Dispersion and Blade Row Interaction in an Axial Compressor. ASME J. Turbomachinery 128 (1), 031009-031009-11.

[46] Hodson, H. \& Howell, R. 2005 Bladerow interactions, transition, and high-lift aerofoils in low-pressure turbines. Аnпи. Rev. Fluid Mech. 37, 7198.

[47] Hunt, J. \& SAVILL, A. 2005 Guidelines and criteria for the use of turbulence models in complex flows, p. 291343. Cambridge University Press.

[48] Ishinara, T., Gotoh, T. \& KanedA, Y. 2009 Study of high-reynolds number isotropic turbulence by direct numerical simulation. Annual Review of Fluid Mechanics 41, 165-180.

[49] IVEngaR, V. \& SANKaR, L. 2012 Comprehensive Application of a First Principles Based Methodology for Design of Axial Compressor Configurations. ASME J. Turbomachinery 134 (6), 061035-061035-9.

[50] Jee, S., Joo, J. \& Medic, G. 2016 Large-eddy simulation of a highpressure turbine vane with inlet turbulence. In ASME Turbo Expo 2016: Turbomachinery Technical Conference and Exposition, pp. V02DT44A019V02DT44A019. American Society of Mechanical Engineers.

[51] Jooy, J., Medic, G., Philipsz, D. \& Bosez, S. 2014 Large-eddy simulation of a compressor rotor. In Center for Turbulence Research Proceedings of the Summaer Program 2014.

[52] Juan, D., JichaO, L., Lipeng, G., Feng, L. \& JingYi, C. 2016 The Impact of Casing Groove Location on Stall Margin and Tip Clearance Flow in a Low-Speed Axial Compressor. ASME J. Turbomachinery 138 (12), 121007121007-11.

[53] Kalitzin, G., Wu, X. \& Durbin, P. 2003 DNS of fully turbulent flow in a LPT passage. International Journal of Heat and Fluid Flow 24 (4), 636-644.

[54] KeadLE, K. \& MCQuilling, M. 2013 Evaluation of RANS transition modeling for high lift LPT flows at low Reynolds number. In ASME Turboexpo, GT2013-95069. ASME.

[55] Kim, J., Moin, P. \& Moser, R. 1987 Turbulence statistics in Fully Developed Channel Flow at Low Reynolds Number. J. Fluid Mech. 177, 133-166.

[56] Koupper, C., Bonneau, G., Gicquel, L. \& Duchaine, F. 2016 Large 
eddy simulations of the combustor turbine interface: Study of the potential and clocking effects. In ASME Turbo Expo 2016: Turbomachinery Technical Conference and Exposition, pp. V05BT17A003-V05BT17A003. American Society of Mechanical Engineers.

[57] De Laborderie, J., Duchaine, F. \& Gicquel, L. 2017 Analysis of a high-pressure multistage axial compressor at off-design conditions with coarse large eddy simulations. In Proceedings of 12th European Conference on Turbomachinery Fluid Dynamics \& Thermodynamics, ETC12,.

[58] Laskowski, G., Kopriva, J., Michelassi, V., Shankaran, S., Paliath, U., Bhaskaran, R., Wang, Q., Talnikar, C., Wang, Z. \& JIA, F. 2016 Future directions of high fidelity cfd for aerothermal turbomachinery analysis and design. In 46th AIAA Fluid Dynamics Conference, p. 3322.

[59] Laurent, C., Mary, I., Gleize, V., Lerat, A. \& ARnal, D. 2011 DNS database of a transitional separation bubble on a flat plate and application to RANS modeling validation. Computers \& Fluids 61, 21-30.

[60] LeACH, K. 1983 Energy Efficient Engine High Pressure Turbine Component Rig Performance Test Report NASA PWA-559-243. Tech. Rep..

[61] LEE, M. \& MOSER, R. 2015 Direct numerical simulation of turbulent channel flow up to $R e_{\tau} \approx 5,200 . J$. Fluid Mech. 774, 395-415.

[62] Leggett, J., Priebe, S., Shabbir, A., Michelassi, V., Sandberg, R. \& RichaRdSON, E. 2018 LES Loss Prediction in an Axial Compressor Cascade at Off-Design Incidences with Free Stream Disturbances. ASME J. Turbomachinery 7 (140), 071005.

[63] LeLE, S. 1997 Computational Aeroacoustics: A Review. AIAA Paper 97-18.

[64] Lengani, D., Simoni, D., Pichler, R., Sandberg, R., Michelassi, V. \& BERTINI, F. 2018 Identification and quantification of losses in a LPT cascade by POD applied to LES data. International Journal of Heat and Fluid Flow 70, 28-40.

[65] Lengani, D., Simoni, D., Ubaldi, M., Zunino, P. \& Bertini, F. 2017 A POD-based procedure for the split of unsteady losses of an LPT cascade. International Journal of Turbomachinery, Propulsion and Power 2 (4), 17.

[66] LeschZiner, M. 2015 Statistical turbulence modelling for fluid dynamicsdemystified: an introductory text for graduate engineering students. World Scientific.

[67] Ling, J., Jones, R. \& Templeton, J. 2016 Machine learning strategies for systems with invariance properties. J. Comp. Phys. 318, 22-35.

[68] Ling, J., KurZawski, A. \& Templeton, J. 2016 Reynolds averaged turbulence modelling using deep neural networks with embedded invariance. $J$. Fluid Mech. 807, 155-166.

[69] Ling, J. \& Templeton, J. 2015 Evaluation of machine learning algorithms for prediction of regions of high Reynolds averaged Navier Stokes uncertainty. Phys. Fluids 27 (8), 085103.

[70] LIU, X. \& RODI, W. 1991 Experiments on transitional boundary layers with wake-induced unsteadiness. J. Fluid Mech. 231, 229-256.

[71] Lumley, J. 1970 Toward a turbulent constitutive relation. J. Fluid Mech. 
41 (2), 413-434.

[72] Mansour, N., Kim, J. \& Moin, P. 1988 Reynolds-stress and dissipationrate budgets in a turbulent channel flow. J. Fluid Mech. 194, 15-44.

[73] Marconcini, M., Pacciani, R., Arnone, A., Michelassi, V., PichLER, R., ZHAO, Y. \& RD, S. 2018 Les and rans analysis of the end-wall flow in a linear lpt cascade with variable inlet conditions, part ii: Loss generation. In ASME Turbo Expo 2018: Turbomachinery Technical Conference and Exposition, pp. GT2018-76450. American Society of Mechanical Engineers.

[74] Matsuura, K. \& KATO, C. 2006 Large-eddy simulation of compressible transitional cascade flows with and without incoming free-stream turbulence. JSME Intern. J. Series B 49 (3), 660-669.

[75] MAYLE, R. 1991 The 1991 IGTI scholar lecture: The role of laminar-turbulent transition in gas turbine engines. ASME J. Turbomachinery 113 (4), 509-536.

[76] MCMullan, W. \& PAGE, G. 2011 Large Eddy Simulation of a Controlled Diffusion Compressor Cascade. Flow, Turbulence and Combustion 86 (2), 207-230.

[77] McNulty, G., Decker, J., Beacher, B. \& Khalid, S. 2004 The Impact of Forward Swept Rotors on Tip Clearance Flows in Subsonic Axial Compressors. ASME J. Turbomachinery 126 (4), 061013-061013-12.

[78] Medic, G. \& Sharma, O. 2012 Large-eddy simulation of flow in a lowpressure turbine cascade. In ASME Turboexpo, GT2012-68878. ASME.

[79] Medic, G., Sharma, O., Jongwook, J., Hardin, L., McCormick, D., Cousins, W., Laurie, E., Shabbir, A., Holley, B. \& Van Slooten, P. 2014 High Efficiency Centrifugal Compressor for Rotorcraft Applications. NASA/CR-2014-218114.

[80] Menter, F. 2009 Review of the shear-stress transport turbulence model experience from an industrial perspective. International Journal of Computational Fluid Dynamics 23 (4), 305-316.

[81] Menter, F. \& Egorov, Y. 2006 SAS turbulence modelling of technical flows. In Direct and Large-Eddy Simulation VI, pp. 687-694. Springer.

[82] MiCHELASSI, V. 1997 Shock-boundary layer interaction and transition modelling in turbomachinery flows. Proceedings of the Institution of Mechanical Engineers, Part A: Journal of Power and Energy 211 (3), 225-234.

[83] Michelassi, V. 2015 Modeling and resolving turbulence in turbomachinery flows. In Tutorial at 2015 ASME Turbo Expo, Montreal, Canada.

[84] Michelassi, V., Chen, L., Pichler, R., SANDberG, R. \& Bhaskaran, R. 2016 High-fidelity simulations of low-pressure turbines: Effect of flow coefficient and reduced frequency on losses. ASME J. Turbomachinery 138 (11), 111006.

[85] Michelassi, V., Martelli, F., Denos, R., Arts, T. \& Sieverding, C. 1999 Unsteady Heat Transfer in StatorRotor Interaction by Two-Equation Turbulence Model. ASME J. Turbomachinery 121 (3), 436-447.

[86] Michelassi, V., Pichler, R., L, C. \& RD, S. 2015 Compressible Direct Numerical Simulation of Low-Pressure Turbines: Part II - Effect of Inflow Disturbances. ASME J. Turbomachinery 137, 071005-1-071005-12.

[87] MiChelASSI, V. \& WissinK, J. 2015 Turbulent kinetic energy production 
in the vane of a low-pressure linear turbine cascade with incoming wakes. International Journal of Rotating Machinery 2015.

[88] Michelassi, V., WissinK, J., FröHLich, J. \& Rodi, W. 2003 Large-eddy simulation of flow around low-pressure turbine blade with incoming wakes. AIAA J. 41 (11), 2143-2156.

[89] Michelassi, V., WissinK, J. \& RODI, W. 2003 Direct numerical simulation, large eddy simulation and unsteady Reynolds-averaged Navier-Stokes simulations of periodic unsteady flow in a low-pressure turbine cascade: A comparison. Proc. IMechE, Part A: J. Power and Energy 217 (4), 403-411.

[90] Milani, P., Ling, J. \& Eaton, J. 2018 Physical interpretation of machine learning models applied to film cooling flows. In ASME Turbo Expo 2018: Turbomachinery Technical Conference and Exposition, pp. V02AT40A006V02AT40A006. American Society of Mechanical Engineers.

[91] Milani, P., Ling, J., Saez-Mischlich, G., Bodart, J. \& Eaton, J. 2018 A machine learning approach for determining the turbulent diffusivity in film cooling flows. ASME J. Turbomachinery 140 (2), 021006.

[92] Moin, P. \& MAHESH, K. 1998 DIRECT NUMERICAL SIMULATION: A Tool in Turbulence Research. Ann. Rev. Fluid Mech. 30 (1), 539-578.

[93] Morata, E., Gourdain, N., Duchaine, F. \& Gicquel, L. 2012 Effects of free-stream turbulence on high pressure turbine blade heat transfer predicted by structured and unstructured LES. International Journal of Heat and Mass Transfer 55 (21-22), 5754-5768.

[94] Nagabhushana, R. \& JefFerson-Loveday, R, T. P. G. L. S. 2014 Large eddy simulations in turbines: Influence of roughness and free-stream turbulence. Flow, Turbulence and Combustion 92, 543-561.

[95] OKui, H., Verstraete, T., Van den Braembussche, R. \& Alsalihi, Z. 2013 Three-Dimensional Design and Optimization of a Transonic Rotor in Axial Flow Compressors. ASME J. Turbomachinery 135 (3), 031009-03100911.

[96] Pacciani, R., Marconcini, M., Arnone, A. \& Bertini, F. 2014 Predicting high-lift low-pressure turbine cascades flow using transition-sensitive turbulence closures. ASME J. Turbomachinery 136 (5), 051007.

[97] Papadogiannis, D., Wang, G., Moreau, S., Duchaine, F., Gicquel, L. \& NicOUD, F. 2016 Assessment of the indirect combustion noise generated in a transonic high-pressure turbine stage. Journal of Engineering for Gas Turbines and Power 138 (4), 041503.

[98] Parish, E. \& Duraisamy, K. 2016 A paradigm for data-driven predictive modeling using field inversion and machine learning. J. Comp. Phys. 305, 758 774.

[99] Parneix, S., Laurence, D. \& Durbin, P. 1998 A procedure for using dns databases. Journal of Fluids Engineering 120 (1), 40-47.

[100] Patel, V., Rodi, W. \& Scheuerer, G. 1985 Turbulence models for nearwall and low Reynolds number flows-a review. AIAA J. 23 (9), 1308-1319.

[101] Pichler, R., Kopriva, J., Laskowski, G., Michelassi, V. \& SandBERG, R. 2016 Highly resolved les of a linear hpt vane cascade using structured and unstructured codes. In ASME Turbo Expo 2016: Turbomachinery 
Technical Conference and Exposition, pp. V02CT39A041-V02CT39A041. American Society of Mechanical Engineers.

[102] Pichler, R., Michelassi, V., Sandberg, R. \& Ong, J. 2017 Highly Resolved LES Study of Gap Size Effect on Low-Pressure Turbine Stage. ASME J. Turbomachinery 140 (2), 021003.

[103] Pichler, R., Sandberg, R., Laskowski, G. \& Michelassi, V. 2017 High-fidelity simulations of a linear hpt vane cascade subject to varying inlet turbulence. In ASME Turbo Expo 2017: Turbomachinery Technical Conference and Exposition, pp. V02AT40A001-V02AT40A001. American Society of Mechanical Engineers.

[104] Pichler, R., Sandberg, R. \& Michelassi, V. 2016 Assessment of grid resolution requirements for accurate simulation of disparate scales of turbulent flow in low-pressure turbines. In ASME Turbo Expo 2016: Turbomachinery Technical Conference and Exposition, pp. V02CT39A030-V02CT39A030. American Society of Mechanical Engineers.

[105] Pichler, R., SAndberg, R., Michelassi, V. \& Bhaskaran, R. 2015 Investigation of the accuracy of RANS models to predict the flow throuh a low pressure turbine. In Proceedings of the ASME - GT2015-43446, pp. 1-14.

[106] Pichler, R., Zhao, Y., RD, S., Michelassi, V., Pacciani, R., MarCONCINI, M. \& ARNONE, A. 2018 Les and rans analysis of the end-wall flow in a linear lpt cascade with variable inlet conditions, part i: Flow and secondary vorticity fields. In ASME Turbo Expo 2018: Turbomachinery Technical Conference and Exposition, pp. GT2018-76233. American Society of Mechanical Engineers.

[107] POPE, S. 1975 A more general effective-viscosity hypothesis. J. Fluid Mech. 72 (2), 331-340.

[108] Postl, D., Balzer, W. \& FAsel, H. 2011 Control of laminar separation using pulsed vortex generator jets: direct numerical simulations. J. Fluid Mech. 676, 81-109.

[109] PrZytarski, P. \& Wheeler, A. 2018 Accurate predictions of loss using high-fidelity methods. In ASME Turbo Expo 2018: Turbomachinery Technical Conference and Exposition, pp. GT2018-77125. American Society of Mechanical Engineers.

[110] Ranjan, R., Deshpande, S. \& Narasimha, R. 2017 New insights from high-resolution compressible dns studies on an lpt blade boundary layer. Computers \& Fluids 153, 49-60.

[111] Raverdy, B., Mary, I., Sagaut, P. \& Liamis, N. 2003 High-resolution large-eddy simulation of flow around low-pressure turbine blade. AIAA J. 41 (3), 390-397.

[112] Rizzetta, D. \& Visbal, M. 2005 Numerical simulation of separation control for transitional highly loaded low-pressure turbines. AIAA J. 43 (9), 19581967.

[113] RoDI, W. 1976 A new algebraic relation for calculating the reynolds stresses. In Gesellschaft Angewandte Mathematik und Mechanik Workshop Paris France, , vol. 56.

[114] Rodi, W., Mansour, N. \& Michelassi, V. 1993 One-equation near-wall 
turbulence modeling with the aid of direct simulation data. J. Fluids Engineering 115 (2), 196-205.

[115] RotTA, J. 1979 A family of turbulence models for three-dimensional boundary layers. In Turbulent Shear Flows I, pp. 267-278. Springer.

[116] Roy, P., Segui, L., Jouhaud, J. \& GicQuel, L. 2017 Resampling strategies to improve surrogate model-based uncertainty quantification: Application to LES of LS89. International Journal for Numerical Methods in Fluids .

[117] Sandberg, R., Pichler, R., Chen, L., Johnstone, R. \& Michelassi, V. 2015 Compressible Direct Numerical Simulation of Low-Pressure Turbines: Part I - Methodology. ASME J. Turbomachinery 137, 051011-1051011-10.

[118] Sandham, N. \& SAndberg, R. 2009 Direct numerical simulation of the early development of a turbulent mixing layer downstream of a splitter plate. Journal of Turbulence $\mathbf{1 0}$ (November), N1.

[119] SARKAR, S. 2009 Influence of wake structure on unsteady flow in a low pressure turbine blade passage. ASME J. Turbomachinery 131 (4).

[120] SCHMitT, F. 2007 About Boussinesq's turbulent viscosity hypothesis: historical remarks and a direct evaluation of its validity. Comptes Rendus Mecanique 335 (9-10), 617-627.

[121] Schulte, V. \& Hodson, H. 1998 Unsteady Wake-Induced Boundary Layer Transition in High Lift LP Turbines. ASME J. Turbomachinery 120 (1), 28.

[122] Scilllitoe, A., Tucker, P. \& Adami, P. 2017 Numerical investigation of three-dimensional separation in an axial flow compressor: The influence of freestream turbulence intensity and endwall boundary layer state. ASME J. Turbomachinery 139, 021011.

[123] Scilllitoe, A D, T. P. G. A. P. 2018 Large eddy simulation of boundary layer transition mechanisms in a gas-turbine compressor cascade. In ASME Turbo Expo 2018: Turbomachinery Technical Conference and Exposition. American Society of Mechanical Engineers.

[124] Segui, L., Gicquel, L., Duchaine, F. \& Laborderie, J. 2017 Les of the LS89 cascade: Influence of inflow turbulence on the flow predictions. In Proceedings of the twelvth European conference on turbomachinery fluid dynamics and thermodynamics, Stockholm, Sweden.

[125] Shahin, I., Gadala, M., Alqaradawi, M. \& Badr, O. 2015 Large Eddy Simulation for a Deep Surge Cycle in a High-Speed Centrifugal Compressor With Vaned Diffuser. ASME J. Turbomachinery 137 (10), 101007.

[126] Sieverding, C., Arts, T., Denos, R. \& Martelli, F. 1996 Investigation of the Flow Field Downstream of a Turbine Trailing Edge Cooled Nozzle Guide Vane. ASME J. Turbomachinery 118 (2), 291-300.

[127] SMAGORINSKY, J. 1963 General circulation experiments with the primitive equations. I. The basic experiment. Mon. Weather Rev. 91, 99-164.

[128] Smirnov, E., Abramov, A., Ivanov, N., Smirnov, P. \& Yakubov, S. 2004 DNS and RANS/LES-computations of complex geometry flows using a parallel multiblock finite-volume code. In Parallel Computational Fluid Dynamics 2003, pp. 219-226. Elsevier.

[129] Smith, L. 2002 Axial Compressor Aerodesign Evolution at General Electric. 
ASME J. Turbomachinery 124 (3), 321-330.

[130] SondergaARD, R., RiviR, R. \& Bons, J. 2002 Control of low-pressure turbine separation using vortex-generator jets. Journal of Propulsion and Power 18 (4), 889-895.

[131] Spalart, P. 2015 Philosophies and fallacies in turbulence modeling. Progress in Aerospace Sciences 74, 1-15.

[132] Spalart, P. \& Allmaras, S. 1992 A One-Equation Turbulence Model for Aerodynamic Flows. AIAA Paper 92-0439 30th AIAA Aerospace Sciences Meeting, Reno, USA.

[133] Spalart, P. R., Shur, M. L., Strelets, M. K. \& Travin, A. K. 2015 Direct simulation and rans modelling of a vortex generator flow. Flow, Turbulence and Combustion 95 (2-3), 335-350.

[134] Stadtmüller, P. \& Fottner, L. 2001 A Test Case for the Numerical Investigation of Wake Passing Effects on a Highly-Loaded LP Turbine Cascade Blade. ASME Paper GT2001-0311.

[135] Stieger, R. \& Hodson, H. 2005 The unsteady development of a turbulent wake through a downstream low-pressure turbine blade passage. ASME J. Turbomachinery 127 (2), 388-394.

[136] Sundstrm, E., Mihaescu, Giachi, M., Belardini, E. \& Michelassi, V. 2017 Analysis of Vaneless Diffuser Stall Instability in a Centrifugal Compressor. Int. J. Turbomach. Propuls. Power 2 (4).

[137] TAM, C. 1995 Computational Aeroacoustics: Issues and Methods. AIAA J. 33 (10), 1788-1796.

[138] TAYlor, J. \& Miller, R. 2016 Competing Three-Dimensional Machanisms in Compressor Flows. ASME J. Turbomachinery 139 (2), 021009.

[139] Trojak, W., Watson, R. \& Tucker, P. 2018 High-order flux reconstruction on stretched and warped meshes. AIAA Journal 57 (1), 341-351.

[140] TuCKer, P. 2011 Computation of unsteady turbomachinery flows: Part1Progress and Challenges. Progress in Aeropsace Sciences 47, 522-545.

[141] TYACKE, J. \& TUCKER, P. 2015 Future use of large eddy simulation in aeroengines. ASME J. Turbomachinery 137 (8), 081005.

[142] VieIRA, R. \& AZEVEDo, J. 2013 RANS simulations of flows with shock wake-boundary layer interaction. In Proceedings of 51st AIAA Aerospace Sciences Meeting, 07-10 January 2013, Grapevine, Texas.

[143] Vincent, P., Witherden, F., Vermeire, B., Park, J. \& IYer, A. 2016 Towards green aviation with python at petascale. In Proceedings of the International Conference for High Performance Computing, Networking, Storage and Analysis, p. 1. IEEE Press.

[144] Wallin, S. \& Johansson, A. 2000 An explicit algebraic Reynolds stress model for incompressible and compressible turbulent flows. J. Fluid Mech. 403, 89-132.

[145] Wang, G., Sanjose, M., Moreau, S., Papadogiannis, D., Duchaine, F. \& GICQUEL, L. 2016 Noise mechanisms in a transonic high-pressure turbine stage. International Journal of Aeroacoustics 15 (1-2), 144-161.

[146] WAng, M., Freund, J. \& LeLE, S. 2006 Computational prediction of flowgenerated sound. Annu. Rev. Fluid Mech. 38, 483-512. 
[147] Watson, R. \& TuCKeR, P. 2016 Perfectly parallel optimization for cutback trailing edges. AIAA J. 54 (7), 2051-2060.

[148] Weatheritt, J., Pichler, R., Sandberg, R., Laskowski, G. \& Michelassi, V. 2017 Machine learning for turbulence model development using a high-fidelity hpt cascade simulation. In ASME Turbo Expo 2017: Turbomachinery Technical Conference and Exposition, pp. V02BT41A015V02BT41A015. American Society of Mechanical Engineers.

[149] Weatheritt, J. \& SANDberG, R. 2016 A novel evolutionary algorithm applied to algebraic modifications of the RANS stress-strain relationship. $J$. Comp. Phys. 325, 22-37.

[150] WeATHERITT, J. \& SANDBERG, R. 2017 The development of algebraic stress models using a novel evolutionary algorithm. International Journal of Heat and Fluid Flow 68, 298-318.

[151] Weatheritt, J., SAndberg, R., Ling, J., SAez, G. \& Bodart, J. 2017 A Comparative Study of Contrasting Machine Learning Frameworks Applied to RANS Modeling of Jets in Crossflow. In ASME Turbo Expo 2017: Turbomachinery Technical Conference and Exposition. GT2017-63403.

[152] Wheeler, A., Sandberg, R., Sandham, N., Pichler, R., Michelassi, V. \& LASKOWSKI, G. 2016 Direct Numerical Simulations of a High-Pressure Turbine Vane. ASME J. Turbomachinery 138 (7), 071003-1-071003-9.

[153] WiLCOX, D. 1988 Reassessment of the scale-determining equation for advanced turbulence models. AIAA J. 26 (11), 1299-1310.

[154] WISSINK, J. 2003 Dns of separating, low reynolds number flow in a turbine cascade with incoming wakes. International Journal of Heat and Fluid Flow 24 (4), 626-635.

[155] Wissink, J., Michelassi, V. \& Rodi, W. 2004 Heat transfer in a laminar separation bubble affected by oscillating external flow. International Journal of Heat and Fluid flow 25 (5), 729-740.

[156] Wissink, J. \& RODI, W. 2006 Direct numerical simulation of flow and heat transfer in a turbine cascade with incoming wakes. J. Fluid Mech. 569, 209247.

[157] Wissink, J., Zaki, T., Rodi, W. \& Durbin, P. 2014 The Effect of Wake Turbulence Intensity on Transition in a Compressor Cascade. Flow, Turbulence and Combustion 93 (4), 555-576.

[158] Wu, X. \& DURBin, P. 2001 Evidence of longitudinal vortices evolved from distorted wakes in a turbine passage. J. Fluid Mech. 446, 199-228.

[159] Wu, X., Jacobs, R., HunT, J. \& Durbin, P. 1999 Simulation of boundary layer transition induced by periodically passing wakes. J. Fluid Mech. 398, $109-153$.

[160] XIAO, H., Wu, J.-L., WAng, J.-X., Sun, R. \& RoY, C. 2016 Quantifying and reducing model-form uncertainties in Reynolds-averaged Navier-Stokes simulations: A data-driven, physics-informed Bayesian approach. J. Comp. Phys. 324, 115-136.

[161] Yamada, K., Furukawa, M., Tamura, Y., Saito, S., Matsuoka, A. \& NAKAYAMA, K. 2017 Large-Scale Detached Eddy Simulation Analysis of 
Stall Inception Process in a Multistage Axial Flow Compressor. ASME J. Turbomachinery 139 (7), 071002-071002-11.

[162] You, D., Wang, M., Moin, P. \& Mittal, R. 2007 Large-eddy simulation analysis of mechanisms for viscous losses in a turbomachinery tip-clearance flow. J. Fluid Mech. 586, 177-204.

[163] Zaki, T., Wissink, J., Durbin, P. \& Rodi, W. 2009 Direct Computations of Boundary Layers Distorted by Migrating Wakes ina Linear Compressor Cascade. Flow, Turbulence and Combustion 83 (3), 307-322.

[164] Zauner, M., Sandham, N., Wheeler, A. \& SAndberg, R. 2017 Linear Stability Prediction of Vortex Structures on High Pressure Turbine Blades. International Journal of Turbomachinery, Propulsion and Power 2 (2), 8.

[165] Zhang, W., ZoU, Z., QI, L., YE, J. \& WANG, L. 2015 Effects of freestream turbulence on separated boundary layer in a low-Re high-lift LP turbine blade. Computers \& Fluids 109, 1-12.

[166] ZhANG, Z. \& DURAisAmy, K. 2015 Machine learning methods for datadriven turbulence modeling. AIAA Aviation paper 2015-2460.

[167] Zhao, Y., Akolekar, H., Weatheritt, J., Michelassi, V. \& SandBERG, R. 2019 Turbulence model development using CFD-driven machine learning. submitted to Journal of Fluid Mechanics .

[168] ZHAO, Y. \& SANDBERG, R. 2019 Using a new entropy loss analysis to assess the accuracy of RANS predictions of an HPT vane. ASME Turbo Expo 2019: Turbomachinery Technical Conference and Exposition, GT2019-90126 . 


\section{University Library}

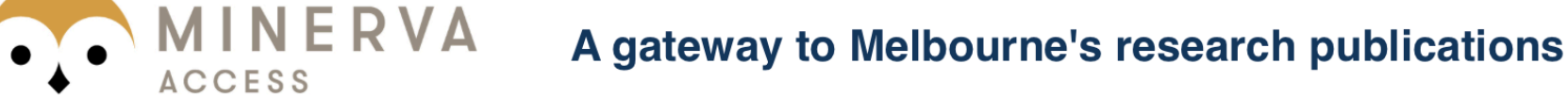

Minerva Access is the Institutional Repository of The University of Melbourne

Author/s:

Sandberg, RD;Michelassi, V

Title:

The Current State of High-Fidelity Simulations for Main Gas Path Turbomachinery Components and Their Industrial Impact

Date:

2019-04-01

Citation:

Sandberg, R. D. \& Michelassi, V. (2019). The Current State of High-Fidelity Simulations for Main Gas Path Turbomachinery Components and Their Industrial Impact. Flow, Turbulence and Combustion, 102 (4), pp.797-848. https://doi.org/10.1007/s10494-019-00013-3.

Persistent Link:

http://hdl.handle.net/11343/241820 